\title{
Temporal Processing in the Visual Cortex of the Awake and Anesthetized Rat
}

\author{
Ida E. J. Aasebø, ${ }^{1}$ - Mikkel E. Lepperød,, ${ }^{1,2}$ Maria Stavrinou, ${ }^{3,4}$ Sandra Nøkkevangen, ${ }^{1}$ Gaute Einevoll, ${ }^{3,5}$ \\ (1DTorkel Hafting, ${ }^{2, *}$ and Marianne Fyhn,
}

DOI:http://dx.doi.org/10.1523/ENEURO.0059-17.2017

${ }^{1}$ Department of Biosciences, University of Oslo, Norway, ${ }^{2}$ Institute of Basic Medical Sciences, University of Oslo, Norway, ${ }^{3}$ Faculty of Science and Technology, Norwegian University of Life Sciences, Norway, ${ }^{4}$ Department of Psychology, University of Oslo, Norway, and ${ }^{5}$ Department of Physics, University of Oslo, Norway

\begin{abstract}
The activity pattern and temporal dynamics within and between neuron ensembles are essential features of information processing and believed to be profoundly affected by anesthesia. Much of our general understanding of sensory information processing, including computational models aimed at mathematically simulating sensory information processing, rely on parameters derived from recordings conducted on animals under anesthesia. Due to the high variety of neuronal subtypes in the brain, population-based estimates of the impact of anesthesia may conceal unit- or ensemble-specific effects of the transition between states. Using chronically implanted tetrodes into primary visual cortex (V1) of rats, we conducted extracellular recordings of single units and followed the same cell ensembles in the awake and anesthetized states. We found that the transition from wakefulness to anesthesia involves unpredictable changes in temporal response characteristics. The latency of single-unit responses to visual stimulation was delayed in anesthesia, with large individual variations between units. Pair-wise correlations between units increased under anesthesia, indicating more synchronized activity. Further, the units within an ensemble show reproducible temporal activity patterns in response to visual stimuli that is changed between states, suggesting state-dependent sequences of activity. The current dataset, with recordings from the same neural ensembles across states, is well suited for validating and testing computational network models. This can lead to testable predictions, bring a deeper understanding of the experimental findings and improve models of neural information processing. Here, we exemplify such a workflow using a Brunel network model.
\end{abstract}

Key words: anesthesia; awake; computational modeling; single units; temporal sequences; visual cortex

\section{Significance Statement}

As investigations of neural information processing have moved from anesthetized to alert animals, a question emerges on how we can compare data and interpret results from recordings from anesthetized animals. Previous efforts to investigate the impact of anesthesia on visual processing have frequently compared population responses from separate animals or experiments. The large diversity of neurons in the cortex demands comparisons within units and ensembles. Here, we followed units across states and found unpredictable and profound differences in the temporal dynamics of single units and ensembles between the anesthetized and awake states. To exemplify the importance of such a comparison, we demonstrate how such data can be used as a basis to test and develop models on network activity.

Received February 23, 2017; accepted July 17, 2017; First published July 24, 2017

The authors declare no competing financial interests.

Author contributions: I.E.J.A., M.E.L., G.E., T.H., and M.F. designed research; I.E.J.A., S.N., M.F., M.E.L., and G.E. performed research; I.E.J.A., M.E.L., T.H., and M.S. analyzed data; I.E.J.A., M.E.L., G.E., T.H., and M.F. wrote the paper.
This work was supported by Norwegian Research Council Grants 204939 (to M.F.) and 231248 (to T.H.) and by the University of Oslo.

${ }^{*}$ T.H. and M.F. are equal senior author contributions.

Acknowledgements: We thank Eivind Nordheim for technical assistance and Dori Derdikman and Rachel M. Thomas for comments and discussion of this manuscript. 


\section{Introduction}

Despite the profound effect of anesthesia on the brain, neurons of the primary sensory cortices continue to respond to adequate stimuli under anesthesia. This has made anesthetized preparations attractive for studying sensory processing and has given insight into the fundamental principles of information processing. Wurtz et al. (1969) reported that neurons in the awake primate visual cortex respond similarly to visual stimuli to those of anesthetized cats and monkeys (Hubel and Wiesel, 1962, 1968). However, during natural vision, the relationship between visual stimulus and activity in visual cortex is less clear, indicating that cortical processing during behavior is heavily influenced by top-down processing and even input from other sensory modalities (lurilli et al., 2012; Zhang et al., 2014). In the visual cortex of rodents, cortical activity is modulated by locomotion as well as behavioral state reflecting fundamental differences in cortical processing between the awake and anesthetized animal (Niell and Stryker, 2008, 2010; Keller et al., 2012; Ayaz et al., 2013). It is well known that anesthesia has profound effects on the brain, e.g., by suppression of neuron and glial cell activity (Greenberg et al., 2008; Schummers et al., 2008; Thrane et al., 2012; Vizuete et al., 2012) and effects on large-scale neuronal networks (Hentschke et al., 2005; Cimenser et al., 2011; Lewis et al., 2012; Pisauro et al., 2013; Bettinardi et al., 2015; Durand et al., 2016). Most of this previous work has been interexperimental comparisons with few experiments following the neurons between states. Consequently, less is known about how the activity of individual neurons in a cell ensemble is affected by the state transition between wakefulness and anesthesia. Considering the large diversity of neuronal subtypes in the cortex, investigations comparing separate populations of neurons recorded in each state can conceal how individual units in a population respond to the transition, since different neurons are sampled in the two populations.

Population activity may be unstructured (Kerr et al., 2005) or can be composed of default activity patterns (Luczak and MacLean, 2012). Neurons in auditory and somatosensory cortices fire in a sequential order during both spontaneous and sensory evoked activity (Luczak et al., 2007, 2009) perhaps reflecting common constraints from the cortical architecture (Luczak and MacLean, 2012). It remains unclear if such patterns are affected by anesthesia, since no previous investigations have followed the same population with single-unit recordings between states in the visual cortex. Moreover, the effect of anesthesia on stimulus-evoked latencies (Pisauro et al., 2013; Durand et al., 2016) may impact the temporal precision of responses and potentially the sequential pat-

Correspondence should be addressed to Marianne Fyhn, Centre for Integrative Neuroplasticity, Department of Biosciences, University of Oslo, Oslo, NO-0361, Norway. E-mail: marianne.fyhn@ibv.uio.no.

DOI:http://dx.doi.org/10.1523/ENEURO.0059-17.2017

Copyright (C) 2017 Aasebø et al.

This is an open-access article distributed under the terms of the Creative Commons Attribution 4.0 International license, which permits unrestricted use, distribution and reproduction in any medium provided that the original work is properly attributed. terns of activation. Temporal precision in neuronal firing is a characteristic of cortical activity in awake and behaving animals (Lee et al., 2005; Siapas et al., 2005) and of activity in the visual system (Mainen and Sejnowski, 1995; Reinagel and Reid, 2002), but how the temporal dynamics of ensembles of neurons in the visual cortex is affected by the transition from wakefulness to anesthesia, remains unresolved.

Using chronically implanted tetrodes in the rat visual cortex we conducted extracellular recordings of single units and local field potentials (LFPs) in response to visual stimulation and followed the same units during behavior, anesthesia, and after recovery from anesthesia. This method separates single units and can, with high temporal precision, follow their activity under different experimental conditions. In contrast to previous work comparing the transition between the awake and anesthetized states (Bayer, 2008; Greenberg et al., 2008) using calcium imaging of $L 2 / 3$ visual cortex neurons, our approach have higher temporal dimension, relate changes to visual stimulation and cover unit sampling across all cortical depths. Furthermore, since the effects of anesthesia can be agent and area specific (Vahle-Hinz and Detsch, 2002), we compare three commonly used anesthetic agents to uncover the effects of anesthesia on unit activity in the visual cortex.

We quantified properties of the temporal activity of single units in local populations between wakefulness and anesthesia. Taken together, this compilation of results show that anesthesia alters temporal dynamics of neural ensembles in the visual cortex. Using a computational approach we further explored the hypothesis that increased inhibition in the network underlies the observed changes from awake to anesthetized states. With the standard and well-established Brunel-type network model consisting of leaky integrate-and-fire neurons (LIF; Brunel, 2000) we find that several of the salient experimental observations regarding differences between the awake and anesthetized states are qualitatively reproduced in the model.

\section{Materials and Methods}

\section{Animals}

Thirteen adult male Long Evans rats were bilaterally implanted with bundles of 16 wire electrodes in tetrode configuration (four tetrodes per hemisphere; Axona) for chronic recordings of neuronal activity in the primary visual cortex. Activity was recorded from the same units before, during, and after anesthesia while presenting visual stimuli. The same units were followed over the course of behavioral states for direct comparisons between states. After surgery, the animals were housed individually in transparent Plexiglas cages (35 × $40 \times 40 \mathrm{~cm})$ and provided with food and water ad libitum. They were kept on a 12/12 h light/dark cycle and testing occurred during the dark phase. All animal procedures were performed in accordance with guidelines from the Norwegian Animal Welfare Act and the European Convention for the Protection of Vertebrate Animals used for Experimental and Other Scientific Purposes. 


\section{Electrode implantation}

The rats were anesthetized with Isoflurane (1-3\%, adjusted to obtain the appropriate surgical depth of anesthesia). Depth of anesthesia was assessed by monitoring heart rate and $\mathrm{O}_{2}$ saturation (\%) in the blood (MouseSTAT, Kent Scientific). Body temperature was monitored and maintained at $37^{\circ} \mathrm{C}$ using homeothermic control system (Kent Scientific). The absence of withdrawal reflex after toe pinch was routinely checked. Analgesia during the procedure was secured by use of presurgically subcutaneous injections of temgesic $(5 \mathrm{mg} / \mathrm{kg})$ and Marcain adrenaline $(0.14 \mathrm{ml} / \mathrm{kg})$. Eyes were kept moist and protected by covering them in a layer of silicone oil. After Isoflurane induction the rats were fixed in a stereotaxic frame and the skull was exposed. Two bilateral craniotomies (1 $\mathrm{mm}$ in diameter) were drilled after vertical and horizontal alignment (according to bregma and lambda) of the head. The transverse sinus was visualized for accurate anterior-posterior coordinates. After a small incisions in the dura mater, microdrives with four tetrodes were shallowly implanted, one in each hemisphere, into the primary visual cortex, $\sim 300 \mu \mathrm{m}$ below the dura mater at an angle of 30-40 degrees in the lateral to medial direction. Coordinates of implantations: $2.5-3.0 \mathrm{~mm}$ anterior to the midpoint of the transverse sinus and 4.8-5.2 lateral to the midline. The electrodes were secured to a microdrive (Axona). Seven small jeweler screws were attached to the skull and several applications of dental cement were used to fix the microdrives into place. Two of the screws were attached to reference electrodes on the microdrives, grounding each of the drives to the skull. To alleviate pain and minimize infections postsurgically, the rats were administered with rimadyl $(5 \mathrm{mg} / \mathrm{kg})$, penicillin $(13.2 \mathrm{mg} / \mathrm{kg})$, and convenia $(8 \mathrm{mg} / \mathrm{kg})$ on the three days following surgery.

\section{Behavior}

During the awake recordings, the rats were allowed to move freely in a square glass enclosure $(28 \times 28 \times 35$ $\mathrm{cm})$, with LCD monitors presenting the visual stimulus covering all four side walls. The monitors covered the rat visual field. The surrounding room was dark, thus the only visible stimulus to the rats was the stimulus presented on the screens. Numerous subsequent recordings with varied activity level were kept, such that each cell had recordings with maximum activity and minimum activity level. The rat's activity level was monitored by tracking (rate, $50 \mathrm{~Hz}$ ) the position of an infrared-light diode attached to the implant and correlated to neural activity recorded simultaneously. During the experimental sessions the animals were monitored closely and the session was discarded if the rat performed any grooming behavior or displayed signs of drowsing/sleeping.

To compare the neuron's activity patterns in the awake and anesthetic states, awake recordings were immediately followed by recordings during anesthesia. The animals were anesthetized using three different regimes: Isoflurane only (1.5\% which corresponds to the minimum alveolar concentration for adult rats; Mazze et al., 1985), Isoflurane with premedication of Dormicum (Isoflurane,
$1 \%$; Midazolam "Dormicum." $1 \mathrm{mg} / \mathrm{kg}$ ) or injections of a mixture of Ketamine and Xylazine $(100 \mathrm{mg} / \mathrm{kg}$ Ketamine and $5 \mathrm{mg} / \mathrm{kg}$ Xylazine). The Isoflurane and Isoflurane/ Dormicum conditions were initially tested with pilot studies to determine the lightest possible level of anesthesia. The Ketamine/Xylazine dose was chosen to match that used in Greenberg et al. (2008). Heart rate, blood oxygen saturation, and the LFP were continuously monitored. An effort was made to keep the animal as lightly anesthestized as possible in the Isoflurane conditions, adjusting the Isoflurane concentration according to the changes in certain physiologic parameters, such as reduced breathing rate, lower heartrate and presence of very low delta frequencies in the LFP. To avoid waking the rat from the light anesthesia, the loss of righting reflex was used to define the rat as unconscious instead of the toe pinch withdrawal reflex. Electrophysiological recordings were initiated 5 min after Isoflurane induction and $10 \mathrm{~min}$ after the injectable anesthetics. Before anethestic induction, droplets of tropicamide were applied to the eyes to widen the pupils. The eyelids were opened by applying a wax strip to the fur below the eyes and pulling the skin down. The head of the rat was placed $21 \mathrm{~cm}$ away from the screen on a raised platform, ensuring that the majority of the rats visual field was covered by the screen. Neural activity was recorded shortly after the anesthesia wore off (10-30 min after, recovery 1) and $24 \mathrm{~h}$ after anesthesia induction (recovery 2).

\section{Electrophysiology}

Tetrodes for microdrives were prepared as described in Csicsvari et al. (1999) using 17- $\mu \mathrm{m} \mathrm{HM-L} \mathrm{coated} \mathrm{iridium/}$ platinum electrode wire (California Fine Wire) twisted to form bundles of four electrodes. The tips of the electrodes were electroplated with platinum to reduce impedances to $100-200 \mathrm{k} \Omega$ at $1 \mathrm{kHz}$. Electrode arrays in the form of tetrodes can reliably be used to isolate several single units per tetrode by comparing the millisecond precise trace of the waveforms occurring on each electrode (Henze et al., 2000). The recorded signal from each electrode was amplified $(5000-18,000 \times)$ band-pass filtered $(0.8-6.7 \mathrm{kHz})$. Spikes were stored at $48 \mathrm{kHz}$ (50 samples per wave form, eight bits per sample) using a 32-bit time stamp (96 Hz clock rate). The LFP was recorded single ended from one of the electrodes, low-pass filtered $(500 \mathrm{~Hz})$ and amplified 1000-2000x.

The recorded units from the electrodes were included without regard for their visual responsiveness, thus sampling nonselectively. All units that could be well isolated and displayed a consistent wave form and position signature throughout the recording period were included in the analyses. The depth-adjustable microdrive was lowered at increments of $50 \mu \mathrm{m}$ to provide sampling cells from deeper cell layers. The tracks of the tetrodes were visualized histologically for anatomic position.

\section{Histology}

At the end of experiments, the rats were anesthetized with pentobarbital $(50 \mathrm{mg} / \mathrm{kg})$ and perfused intracardially with $0.9 \%$ saline and $4 \%$ formaldehyde. The brains were stored in formaldehyde and placed in a $30 \%$ sucrose 
solution for $72 \mathrm{~h}$ before sectioning by a cryostat. Coronal sections $(40 \mu \mathrm{m})$ were cut, mounted on glass slides, and stained for Nissl bodies with cresyl violet (Sigma-Aldrich). The tetrode tracks were measured and imaged with a light microscope (Axioplan microscope, Axiocam HRZ camera, AxioVision software and MosaiX, Zeiss). All electrode traces were verified to be localized within the visual cortex, based on cytoarchitectonic criteria. The recording location was extrapolated from deepest trace identified by histologic inspection of the sections and the tetrodeturning log. Shrinkage of the tissue was adjusted for.

\section{Spike sorting}

Offline spike sorting was performed using graphical cluster-cutting software (Tint, Axona). Triggered spikes were assigned to clusters via the method of cluster cutting (Wilson and McNaughton, 1993; Skaggs et al., 1996), where a cell cluster is isolated from noise and other clusters on the basis of spike wave-shape and amplitude. Several evaluations of cluster quality were performed. First, a requirement of each cell cluster was that interspike interval histograms revealed few $(<0.1 \%)$ or no spikes occurring within $2 \mathrm{~ms}$ of one another (Bruno and Simons, 2002). Second, the clusters need to be visibly isolated from the others and the separation distance between the clusters was quantified by calculating Mahalonobis distance (Harris et al., 2000). Third, the presence of a "common refractoriness," i.e., an absence of spikes in the refractory period of two overlapping units, was used as an indication that two clusters belong to the same unit (Fee et al., 1996). Fourth, all cluster identification needed to clearly persist between the awake, anesthesia, and recovery states. Any ambiguity resulted in unit exclusion.

\section{Visual stimuli}

The visual stimuli were presented on four monitors (Dell, Ultrasharp, $29 \times 36 \mathrm{~cm}, 60-\mathrm{Hz}$ refresh rate, mean luminance $70 \mathrm{~cd} / \mathrm{m}^{2}$ ) positioned in a square closely surrounding the glass enclosure in the awake condition. The monitors were between 7-32 cm away from the rats head as he moved within the box, and distended $29 \mathrm{~cm}$ in height above him on all sides thus covering the rats visual space. In anesthesia the rat was placed $21 \mathrm{~cm}$ away from three of the screens, covering the visual field. The Psychophysics Toolbox extension in Matlab (Brainard, 1997; Pelli, 1997) was used to provide the visual stimuli, which consisted of drifting sinusoidal gratings where each orientation was presented for $0.5 \mathrm{~s}$ with a 0.5 -s blank gray screen between each orientation. Eight orientations were used and the stimuli were repeated twelve times for each session with the orientation sequence randomly distributed. The Psychophysics toolbox program in Matlab was modified to report millisecond precise timestamps to the recording software on a separate computer. This ensured that initiation and termination of each stimulus was detected by the recording system and produced highly temporally accurate spike/stimulus data.

The same stimuli parameters were used throughout the majority of the recordings, to keep the stimulation paradigm conditions comparable throughout $1 \mathrm{~h}$ of anesthesia, and between anesthetic regimes. Previous findings in acute recordings show that the spatial frequency eliciting the most responses from neurons in the rat visual cortex is $0.08 \mathrm{c} / \mathrm{d}$ and the optimal response for temporal frequency has been found to be between 3.44 and $6.88 \mathrm{~Hz}$ (Girman et al., 1999). The spatial and temporal specificity was set to $0.08 \mathrm{c} / \mathrm{d}$ and $4 \mathrm{~Hz}$, respectively, for the majority of the experiments $(n=205)$. The measures of spatial and temporal frequencies were performed on a separate population of units $(n=68)$. For testing of spatial frequencies the temporal frequency was kept fixed at $4 \mathrm{~Hz}$ while the spatial frequencies used were $0.02,0.04,0.08,0.16$, and $0.3 \mathrm{c} / \mathrm{d}$. When testing the temporal frequencies the spatial frequency was kept fixed at $0.08 \mathrm{c} / \mathrm{d}$ while the temporal frequencies presented were 2,4 , and $8 \mathrm{~Hz}$. The order of the frequency presentations were randomly shifted between experiments. And all visual frequencies were tested twice per state to minimize individual trial variations. Although the movement of the rat allowed its specific spatial frequency to vary between 0.04 and $0.12 \mathrm{c} / \mathrm{d}$, only sessions where the animal was sessile throughout the whole trial were used. In other words, no spatial frequency variance occurred within one trial. Furthermore, the maximum spatial frequency change possible was calculated and found to be limited to one spatial frequency group (e.g., $0.02-0.04$ or $0.04-0.08 \mathrm{c} / \mathrm{d}$ ). Finally, the spatial frequency extremes such as low versus high frequencies remain unaffected by the impact of any potential movement.

\section{Data analysis}

All analyses were, unless stated otherwise, conducted on averages of two recordings performed after 30 and 40 min of anesthesia to minimize influence of the fluctuating effect of anesthetic induction. The calculation of overall firing rates was performed as a general measure including both spontaneous and evoked time periods. The separation of the putative interneuron pool followed the same procedure as in Barthó et al. (2004) where units were classified according to their wave form properties. Two parameters were used to isolate the putative interneurons (narrow spiking) from the putative excitatory (broadspiking) neurons: the relative time from trough to peak in $\mathrm{ms}$ and the width of the wave form at half amplitude of the peak. These parameters were found by Barthó et al. (2004) to produce a reliable separation of the cell classes. Fitting the data with two 2-D Gaussians resulted in a bimodal clustering of units (Fig. $1 F$ ). To ensure that other cell classes did not interfere with the classification of units, the following units were excluded: units that reached signal saturation, that had a low signal-to-noise ratio (SNR <2.75; Suner et al., 2005; Smith and Kohn, 2008), or units that showed the triphasic wave form typically associated with axonal activity (Robbins et al., 2013).

Evoked and spontaneous firing rates were investigated by creating peristimulus time histograms (PSTHs) of the combined sessions (repeating and alternating drifting grating and blank screens) and spike trains were convolved with a Gaussian kernel of $10-\mathrm{ms}$ width and a sampling period of $1 \mathrm{~ms}$. Evoked rates was calculated as the maximum firing rate of each unit across all bins following stimulus presentation, and the spontaneous max- 
A
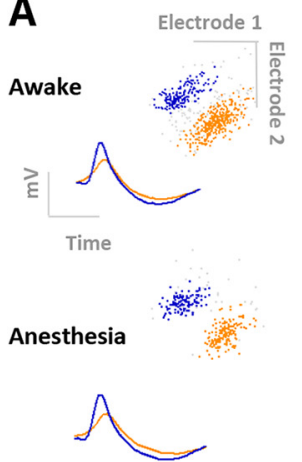

Recovery

$\mathbf{F}$

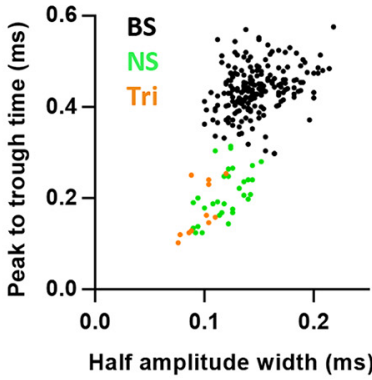

$\mathrm{H}$

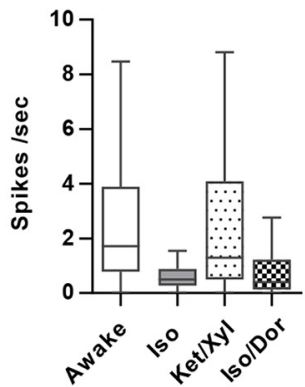

K

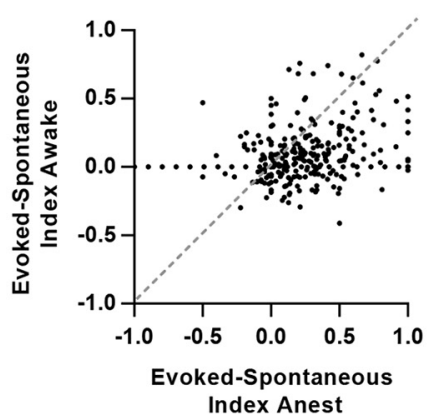

B

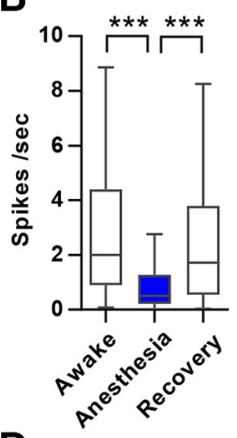

D

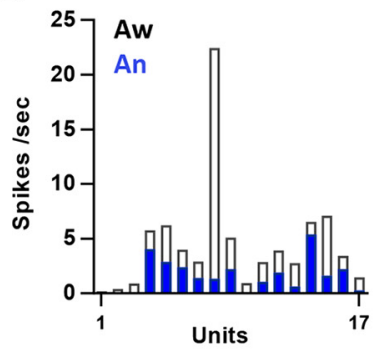

C

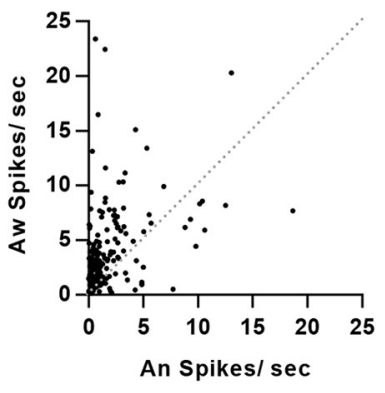

E

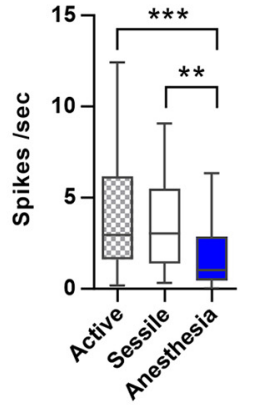

G
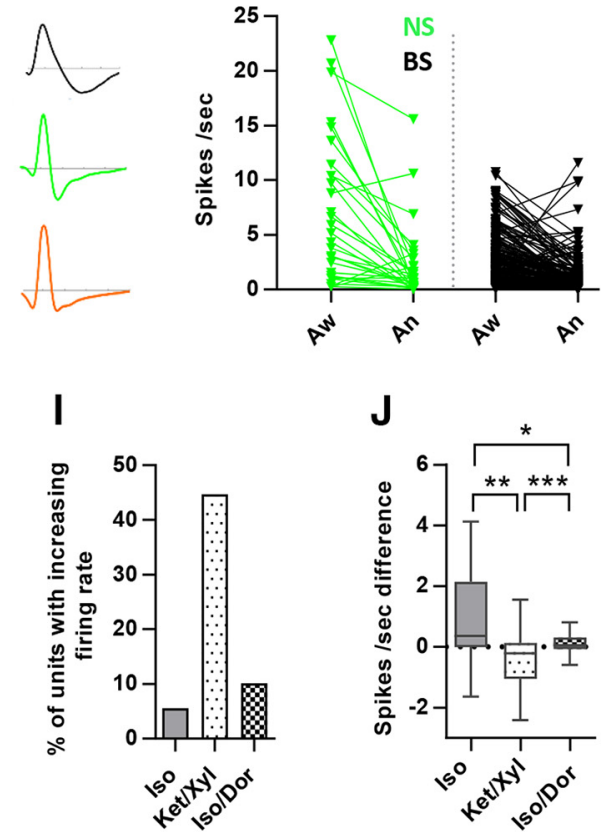

J

$\mathbf{L}$

M
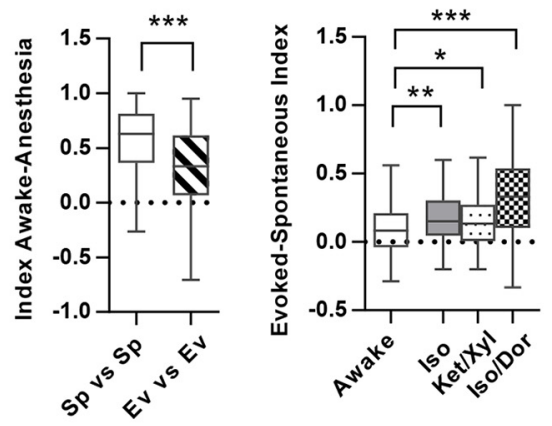

Figure 1. Single unit activity followed between the awake state, anesthesia, and the recovery from anesthesia. $\boldsymbol{A}$, Two example units in all three states, spike waveforms and spike clusters are shown. $\boldsymbol{B}$, Box plot of the firing rates of the units from recording sessions 


\section{continued}

in the awake state, anesthesia, and recovery from anesthesia $(n=193)$. $\boldsymbol{C}$, Scatter plot of the firing rates for all single units in the awake and anesthetic state. $\boldsymbol{D}$, Single ensemble of 17 simultaneously recorded units illustrating within-ensemble variations of firing rate reduction with anesthesia (Isoflurane). $\boldsymbol{E}$, Firing rates in active and sessile sessions relative to anesthesia $(n=51)$. $\boldsymbol{F}$, right panel, Scatter plot of wave form properties of the spikes of all units, $y$-axis represent the time from peak to trough (ms) and the $x$-axis shows the duration (ms) of the peak at half amplitude. Green: NS = narrow spiking units $(n=31)$; black: BS $=$ broad spiking units $(n=209)$; orange: Tri $=$ triphasic units $(n=11)$. Left panel, Example waveforms of BS, NS, and Tri-units. G, Firing rate of narrow spiking units $(n=31)$ versus broad spiking units $(n=209)$ in the awake and anesthetized state. $\boldsymbol{H}$, Firing rates in three different anesthetic regimes: Isoflurane/Dormicum $(n=145)$, Isoflurane $(n=70)$, Ketamine/Xylazine $(n=49)$. I, Percentage of units that respond to anesthesia with an increase in firing rate. $J$, Difference in firing rates between the first 10 and the last 60 min of anesthesia (Iso/Dor $n=109$, Iso $n=$ 65 , Ket/Xyl $n=45)$. K, Raster plot of the evoked- spontaneous index for each unit in the awake and anesthetic state $(n=257)$. $L$, Box plot of indexes calculated on spontaneous activity in both states, and evoked activity in both states for each unit. $\boldsymbol{M}$, Evoked and spontaneous index for units across three anesthetic regimes (Iso $n=70$, Ket/Xyl $n=48$, Iso/Dor $n=133$ ). All box and whiskers plots line show median, upper quartile, lower quartile and whiskers indicate Tukey interquartile range.

imum rates were calculated as the maximum firing rate of each unit across all blank screen bins. The first $200 \mathrm{~ms}$ of the spontaneous period was extracted from this maximum spontaneous rate estimation to exclude cases where units responded to stimulus OFF from the previous drifting gratings presentation. The relationship between evoked and spontaneous firing rates were quantified by an index calculated as (R1 - R2)/(R1 + R2), where R1 is the maximum evoked rate and $\mathrm{R} 2$ the maximum spontaneous rate. For analysis of the contribution of spontaneous versus evoked activity to this index, another index was calculated separately on spontaneous activity in both states and evoked activity in both states for each unit $[(R 1-R 2) /(R 1+R 2)$, spontaneous awake (R1) and spontaneous anesthesia (R2), or evoked awake (R1) and evoked anesthesia (R2)].

The LFP trace was investigated by extracting the time period following each stimulus onset and identifying the latency to each peak and trough in the trace signature. Two response latencies for LFP were calculated as the time from stimulus onset to the first peak and trough above $1.5 *$ SD from the mean of the LFP trace. LFP latencies were calculated per tetrode depth and not per session to avoid errors from oversampling the same position.

Latency from stimulus onset to the peak response rate was investigated by performing Peak detection on the PSTH by calculating the peaks with firing rates that exceeded $1 \mathrm{SD}$ from the mean firing rate. If several peaks after stimulus onset were present, the first peak was selected. To avoid the erroneous detection of random peaks the peak spike rate density needed to exceed $1 \mathrm{~Hz}$ to remove units with low firing rates. To ensure that only visually evoked units were included in the latency calculations, units were excluded if the mean evoked firing rate $( \pm S D)$ did not exceed the mean $( \pm S D)$ for the spontaneous time period. Additionally, a normalized firing rate was calculated per bin per unit, where firing rates of each bin was normalized to the mean firing rate (R2) of each unit (R1) $(\mathrm{R} 1$ - R2)/(R1 + R2). To quantify peak latency onset, the time point where firing rate exceeded $10 \%$ of the baseline to peak range was identified and kept if it remained higher than the baseline for a minimum of $25 \mathrm{~ms}$. Baseline was estimated as the mean response -50 to 50 ms to stimulus onset (Brincat and Connor, 2006).
Pearson correlation coefficients (CCs) between pairs of cells were calculated in bin sizes of $10 \mathrm{~ms}$, and were performed between units from a local population, i.e., from one microdrive in one hemisphere separately. Pairwise correlations were calculated separately for the evoked and spontaneous time period, again the first 200 $\mathrm{ms}$ of the spontaneous time period was removed.

The presence of burst suppression in each session of anesthesia was determined by visual inspection of the LFP trace and time-frequency wavelet analyses (TallonBaudry et al., 1997). To be included in the pool of burst suppression sessions the LFP trace needed to have clearly visible bands of activity with high amplitude over a large frequency spectrum with clear, long-lasting isoelectric periods in between (Swank and Watson, 1949; Ferron et al., 2009).

For the analysis of temporal sequences of population activity (Luczak et al., 2009), a bin size $10 \mathrm{~ms}$ was used for a time period of $200 \mathrm{~ms}$ after stimulus onset. Varying bin size did not affect the conclusions. The details of the method are described in the results section.

To quantify the response derived from the visual stimulus with varied spatial and temporal frequencies we used two techniques. First, in accordance with Niell and Stryker (2008) and Girman et al. (1999), we selected the spatial and temporal frequency with the maximum firing rate for each unit in awake and anesthesia. Second, we computed a normalized firing rate by scaling each unit response(R) in all presented frequencies between one and zero; for this, we used $(\operatorname{maxR}-\mathrm{R}) /(\operatorname{maxR}-\mathrm{minR})$ per unit.

\section{Statistical analysis}

All comparisons were checked for normality with the D'Agostino-Pearson omnibus test and Levenes test for equal variances was performed on the between-groups comparisons; $t$ tests and ANOVA were used for the normally distributed data. Mann-Whitney, Wilcoxon ranksum, Friedman repeated measures, or Kruskal-Wallis tests were performed for non-normal distributions. Dunn's post hoc tests were used for Friedmann and Kruskall Wallis multiple comparisons, while Holm-Sidaks test was used for ANOVA. Box plots show median, upper quartile, lower quartile, and whiskers indicate Tukey interquartile range. All statistical tests used are included in Table 2. 
Table 1. Parameter values for simulations

\begin{tabular}{lll}
\hline Parameter name & Awake & Anesthetized \\
Synaptic delay $(\mathrm{D})$ & $1.5 \mathrm{~ms}$ & \\
Membrane capacitance $(\mathrm{C})$ & $281 \mathrm{pF}$ & \\
Membrane time constant $\left(\tau_{\mathrm{m}}\right)$ & $281 / 20 \mathrm{~ms}$ & \\
Threshold $(\theta)$ & $-50.5 \mathrm{mV}$ & \\
Refractory period $\left(\tau_{\mathrm{rp}}\right)$ & $2 \mathrm{~ms}$ & \\
Equilibrium potential $\left(\mathrm{E}_{\mathrm{L}}\right)$ & $-60 \mathrm{mV}$ & $-65 \mathrm{mV}$ \\
Reset potential $\left(\mathrm{V}_{\mathrm{r}}\right)$ & $-60 \mathrm{mV}$ & $-65 \mathrm{mV}$ \\
Excitatory synaptic efficacy $\left(\mathrm{J}_{\mathrm{E}}\right)$ & $0.5 \mathrm{mV}$ & \\
Relative inhibition $(\mathrm{g})$ & 10.5 & 11 \\
Excitatory connectivity $\left(\varepsilon_{\mathrm{E}}\right)$ & 0.1 & \\
Inhibitory connectivity $\left(\varepsilon_{\mathrm{l}}\right)$ & 0.1 & \\
Number of neurons $(\mathrm{N})$ & 10000 & \\
Excitatory neurons $\left(\mathrm{N}_{\mathrm{E}}\right)$ & 8000 & \\
Cortical input rate $\left(v_{\mathrm{E}, \mathrm{I}, \mathrm{ext}}\right)$ & $0.7 \mathrm{~Hz}$ & \\
Cortical connectivity $(\varepsilon)$ & 0.1 & \\
Thalamic rate $\left(v_{\mathrm{E}, \mathrm{l}, \mathrm{pulse}}\right)$ & $1 \mathrm{~Hz}$ & \\
Thalamic connectivity $(\varepsilon)$ & 0.1 & \\
Thalamic duration & $50 \mathrm{~ms}$ & \\
Trials of thalamic input & 100 & \\
Simulation time & $100 * 1050 \mathrm{~ms}$ & \\
& &
\end{tabular}

\section{Computational modeling}

Neuronal populations in primary visual cortex were represented by a randomly connected network of leaky integrate and fire neurons similarly as in Brunel (2000) with parameters given in Table 1. Cortical input was modeled as Poisson-distributed spikes depolarizing the entire network acting as an excitatory drive. Thalamic input was modeled as Poisson-distributed bursts depolarizing the entire population of inhibitory and excitatory neurons. Strong inhibitory synaptic efficacies were introduced to reproduce the low firing rates found experimentally. To simulate the transition to an anesthetized state we (1) reduced the equilibrium potential of every neuron and (2) increased the inhibitory synaptic efficacy. To investigate how the simulation results were dependent on synaptic efficacy distributions, additional numerical experiments were performed with lognormal distributed synaptic efficacies as defined in NEST (nest-simulator.org; Gewaltig and Diesmann, 2007). These simulations were performed with the same parameter values as the Brunel-type network, where the synaptic efficacies given in Table 1 represents the mean of the lognormal distribution.

\section{Results}

To investigate how anesthesia affects neural activity of units in the visual cortex, we first examined the overall firing rates of units followed between the awake state, anesthesia, and after recovery from anesthesia (Fig. 1A). In the awake state, the average firing rate of all units was $3.28 \mathrm{~Hz}( \pm 0.28 \mathrm{~Hz})$ and decreased to $1.16 \mathrm{~Hz}( \pm 0.13 \mathrm{~Hz})$ during anesthesia (Fig. 1B,C). Within $30 \mathrm{~min}$ after anesthesia, the firing rates were restored to baseline levels $(2.97 \pm 0.28 \mathrm{~Hz}, n=193$; Fig. 1B). The anesthesiamediated decrease in firing rate was significant for both awake versus anesthesia and anesthesia versus recovery ( $p<0.0001, n=193$, Friedman test, Dunn's post hoc). Most cells (220/269 units) reduced their firing rate by $50 \%$ or more in anesthesia, while a small fraction showed increased (39/269 units) or stable $(10 / 269$ units; $<10 \%$ change) firing rates with anesthesia (Fig. 1C). Simultaneous recordings of ensembles of units revealed that neighboring neurons may respond differently to the same level of anesthesia (Fig. 1D). Different intraensemble responses indicate that effects of anesthesia on cortical processing are more easily identified in recordings of the same population of units across states. Sampling was conducted across all cortical layers through the incremental lowering of the tetrodes, with an estimated layer representation of 44 units from L2/3, 36 from L4, 121 from L5 and 54 from L6, and 14 unknown.

To examine if the difference in unit activity between awake and anesthesia may be related to effects of locomotion (Niell and Stryker, 2010; Keller et al., 2012; Ayaz et al., 2013), we monitored the rat's level of activity during recording. The animal's movement was monitored by tracking the position of the head of the rat. Recording sessions were classified as "sessile" or "active" when the path length was shorter than 5 or exceeded $100 \mathrm{~cm} / \mathrm{min}$, respectively. The firing rate was not significantly increased with movement but unit firing rates during in the awake states were both significantly different from that of anesthesia (Fig. 1E, $p=0.0046, n=51$, Friedman test, Dunn's post hoc test). It is likely that our small recording chamber limited movement to such extent that the relation between firing rate and running speed were not evident. Still, to avoid any confounding effects of movement in the awake state, the remaining analyses on the awake state are restricted to the sessile awake state (unless specified otherwise).

\section{Larger impact of anesthesia on narrow spiking units compared to broad spiking units}

Because of their different types of receptors, excitatory and inhibitory neurons are likely to respond differently to the anesthetics. We therefore examined how putative inhibitory and excitatory neurons were affected by anesthesia. Based on the extracellular wave form, neocortical units can be classified into a group with broader spiking waveforms and a more narrow spiking group (McCormick et al., 1985; Bruno and Simons, 2002; Barthó et al., 2004; Niell and Stryker, 2008; lurilli et al., 2013). The narrow spiking group posesses characteristics corresponding to inhibitory interneurons (predominantly fast spiking; McCormick et al., 1985; Barthó et al., 2004), while the broad spiking group is largely dominated by excitatory neurons. Indeed, plotting the wave form properties (Barthó et al., 2004) of peak to trough time versus half-amplitude width of all recorded units revealed a bimodal distribution (Fig. $1 F$ ), in accordance to previous reports (Bruno and Simons, 2002; Barthó et al., 2004; Niell and Stryker, 2008; Sirota et al., 2008; lurilli et al., 2013). Eleven units were excluded from the narrow spiking population as they showed a triphasic wave form which is likely to correspond to axonal activity (Robbins et al., 2013).

The narrow spiking units showed a reduction in average firing rate from $6.80 \pm 1.18 \mathrm{~Hz}$ in the awake state to 2.05 $\pm 0.60 \mathrm{~Hz}(n=31)$ under anesthesia. Calculating the relative change in firing rate for each neuron gives a 
median reduction of $67 \%(p<0.0001, n=31$. Wilcoxon; Fig. 1G). In comparison, the reduction in firing rate for the broad spiking population showed a median of $52 \%$ (awake: $2.56 \pm 0.17 \mathrm{~Hz}$ vs anesthesia: $1.07 \pm 0.11 \mathrm{~Hz}$, $p>0.0001, n=209$; Wilcoxon). The effect of anesthesia on firing rates was significantly greater in the narrow spiking population compared to the broad spiking population (awake-anesthesia difference: $4.75 \pm 1.08 \mathrm{~Hz}, n=$ 31 vs $1.49 \pm 0.16 \mathrm{~Hz}, n=209, p=0.002$, Mann-Whitney; Fig. 1G). The larger impact of anesthesia on fast-spiking inhibitory neurons indicates specific effects on cortical processing during anesthesia.

As the quantification of firing rate reduction performed in Figure $1 B-G$ was performed on all units across all three types of anesthesia as a general comparison, we further wanted to asses how the various types of anesthetics contributed to this average. The GABAergic agonistic anesthestic regimes (Isoflurane and Isoflurane/Dormicum) resulted in a decrease in firing rate (Iso $p<0.0001, n=$ 145; Iso/Dor $p<0.0001, n=70$; Wilcoxon) while Ketamine/Xylazine did not produce a significant decrease in firing rate versus the awake state $(p=0.867, n=48)$. The largest reduction in firing rate was for units under isoflurane anesthesia and less for Isoflurane/Dormicum (Fig. $1 H, p<0.0001$; Kruskal-Wallis test, Iso/Dor-Iso $p=$ 0.0002 , Iso/Dor-Ket/Xyl $p<0.0001$, Iso-Ket/Xyl $p<$ 0.0001 , Dunn's post hoc test). Furthermore, units in the Ketamine/Xylazine condition had a large percentage of units that increased their firing rate in response to anesthesia (45\%; Fig. 1/). Finally, to test the firing rate stability of the anesthetic regimes, we compared the firing rate change throughout $1 \mathrm{~h}$ of anesthesia. We found that the Isoflurane/Dormicum condition had most stable response dynamics (Fig. 1J, $p<0.0001$; Kruskal-Wallis test, Iso/ Dor vs Iso $p=0.011$, Iso/Dor vs Ket/Xyl $p=0.002$, Iso vs Ket/Xyl $p<0.0001$; Dunn's post hoc test, Iso/Dor $n=$ 109 , Iso $n=65$, Ket/Xyl $n=45$ ). Due to this stability, we conducted our investigation of responses to spatial and temporal frequencies with Isoflurane/Dormicum.

\section{Spontaneous versus evoked activity}

The variable effects on firing rates of individual neurons within a population suggest that information processing of incoming sensory information is affected.

We observed a collective reduction in both spontaneous and stimulus-evoked firing rates during anesthesia. To assess how spontaneous and evoked rates were affected for each unit, we calculated an evoked-spontaneous index for each state $[(R 1-R 2) /(R 1+R 2) R 1$-maximum evoked rate and R2-spontaneous rate]. We found that during anesthesia the units have a higher ratio of evoked to spontaneous activity (Fig. $1 K, p<0.0001$, paired $t$ test, $n=257$ ). Also, by calculating separate indexes on spontaneous activity between states and evoked activity between states for each unit, we found that the relative decrease in spontaneous activity is larger than the relative increase in evoked activity (Fig. $1 L, p<0.0001, n=260$; Wilcoxon). Thus, our results support the proposed effect from previous investigations that an effect of anesthesia on unit activity is mainly a reduction in spontaneous ac- tivity (Niell and Stryker, 2010). This was also true for the three different anesthetic regimes when comparing the evoked-spontaneous index [lso $p=0.0011[n=70]$, Ket/Xyl $p=0.028$ [ $n=48]$, Iso/Dor $p<0.0001$ [ $n=133$ ]; (Fig. 1M)]. Interestingly, the Ketamine/Xylazine condtion produces a change in evoked and spontaneous activity in the units without a significant firing rate depression.

To elucidate if the lower index of spontaneous to evoked rate in the awake state was merely due to movement, we compared sessions when the animal was sessile and actively moving on a subset of units. We found no difference in the evoked-spontaneous index between sessile and moving sessions [mean index moving $=0.016$ $( \pm 0.024)$, mean index sessile $=-0.012( \pm 0.029)$, mean index under anesthesia $=0.141( \pm 0.030)$, moving vs sessile $p=0.6177$, moving vs anesthesia $p=0.0133$, sessile vs anesthesia $p>0.0001$. Friedman, Dunn's post hoc], supporting that the observed reduction of spontaneous activity is explained by anesthesia.

\section{Temporal changes of unit activity in anesthesia}

Temporal structure of neuronal activity is essential for cortical processing but how the timing of unit activity within an ensemble is affected by anesthesia in the visual cortex remains unresolved. We therefore examined how the temporal specificity of units is affected by the change from awake to anesthesia.

\section{Visually evoked latencies are delayed under anesthesia}

Local field potentials (LFPs) provide insight into the cooperative properties of local neuronal populations and reflect synaptic activity from larger populations of neurons compared to the number of active units picked up by the electrodes (Buzsáki, 2006). To investigate the latency in response to visual stimulation, we examined the temporal profile of LFP responses to visual stimuli in the awake and anesthetized states. The average stimulus-triggered LFP signal yielded a signature with a trough followed by a peak for the majority of LFP traces investigated (Fig. $2 A, E$ ). The time to trough was significantly longer in anesthesia compared to awake (Fig. 2B; awake: $92 \pm 14 \mathrm{~ms}$, anesthesia: $112 \pm 37 \mathrm{~ms}, p=0.0048, n=17$; Wilcoxon), as well as the time to peak after trough (awake: $168 \pm 49 \mathrm{~ms}$, anesthesia: $307 \pm 140 \mathrm{~ms}, p=0.003$; Wilcoxon). Interestingly, the delay between trough and peak was also different between states (Fig. 2C; awake: $77 \pm 44 \mathrm{~ms}$, anesthesia: $202 \pm 111 \mathrm{~ms}, p=0.002$; Wilcoxon), i.e., the time to peak has a significantly larger delay than the time to the trough. Thus, the LFP signature is not only shifted in time, but appears to last longer during anesthesia. Finally, we find a large difference in amplitude of the first peak in the LFP signature (Fig. $2 D, p<0.0001$; Wilcoxon). Figure $2 E$ shows the average LFP trace across all experiments (top panel) as well as the response in each experimental session (bottom panel) to highlight the lack of variability between the traces. To avoid oversampling and biasing the LFP analysis, only one LFP trace per anesthesia recording session was included (17 sessions, 9 rats).

To compare how response latencies related to unit activity, we analyzed the temporal response profile of 
LFP

A

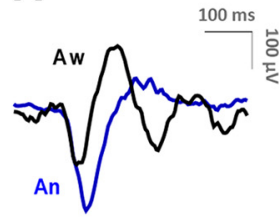

Vis. stimuli

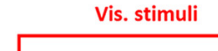

Single units
B

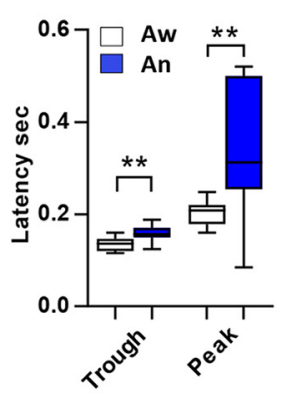

C

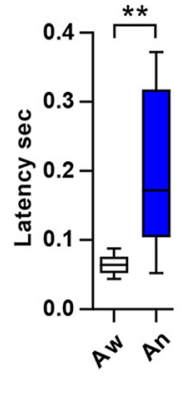

D

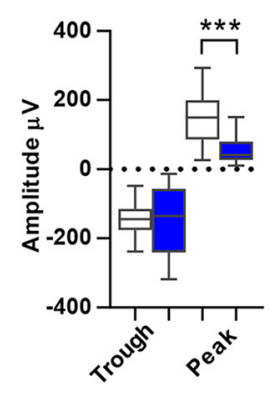

Vis. stimuli

E
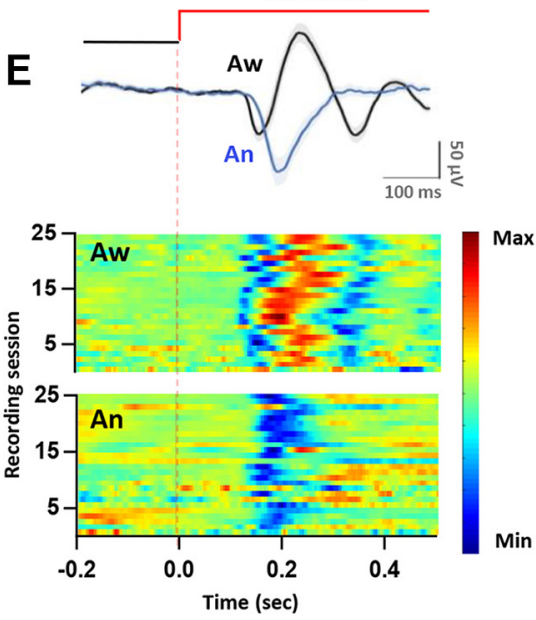

\section{F Awake}
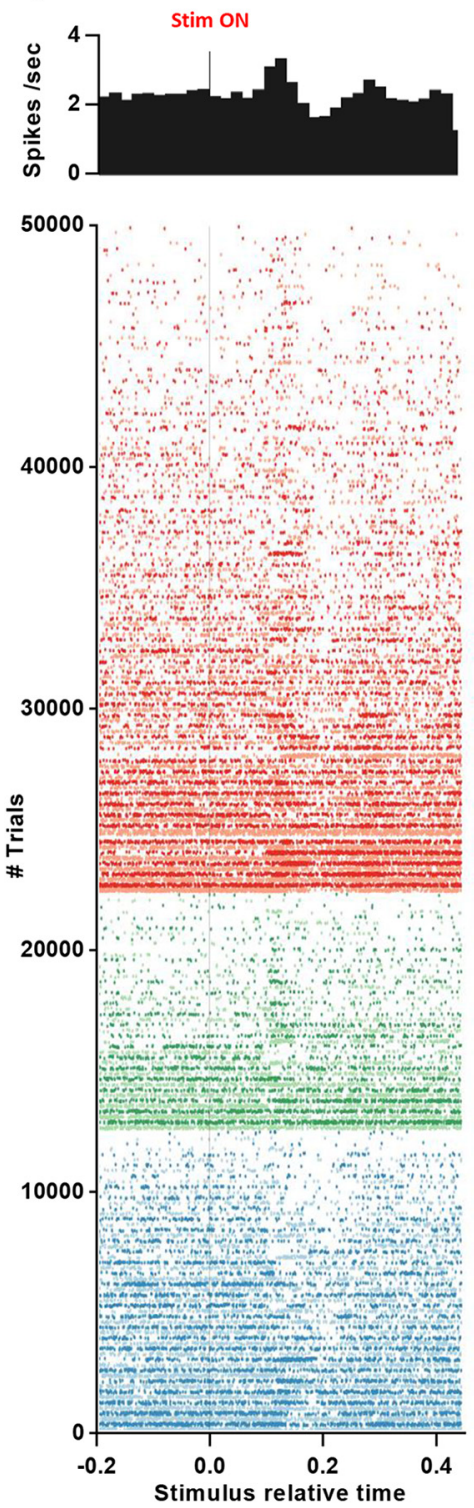

Anesthesia
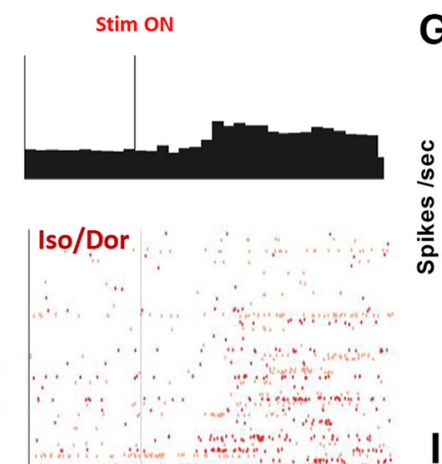

G
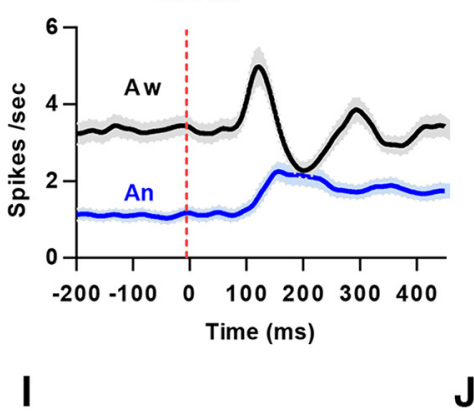

H

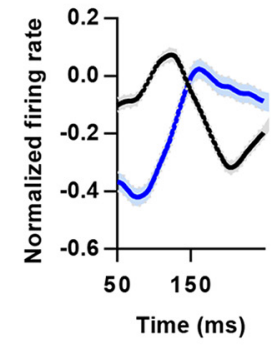

J

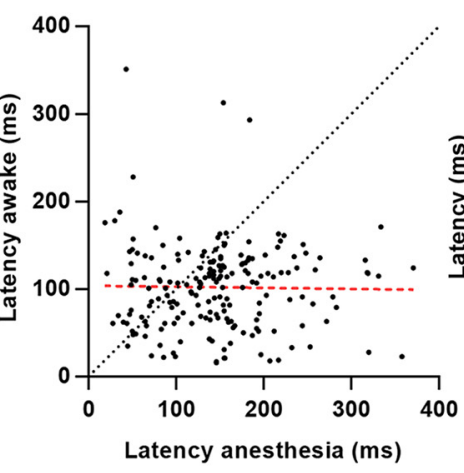

K

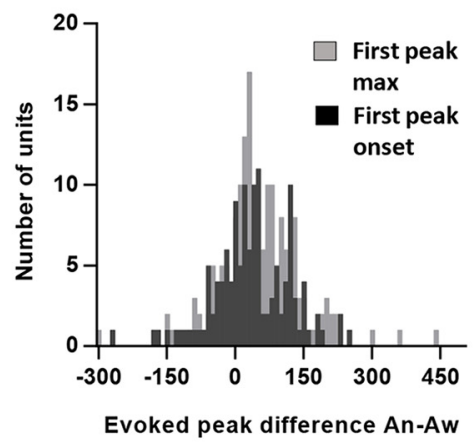

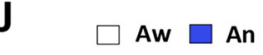

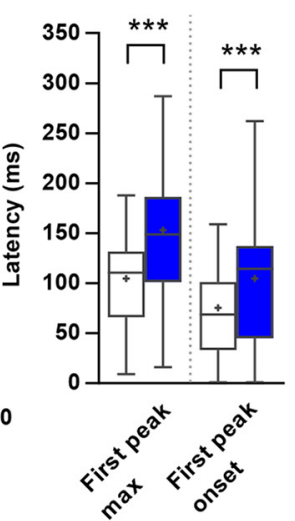

L

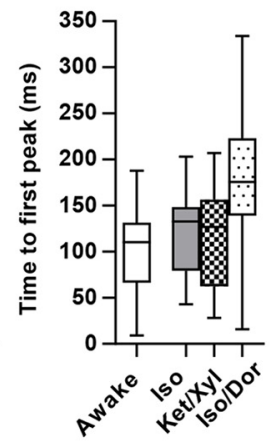

Figure 2. Evoked response latencies of the LFP and single units followed from the awake state to anesthesia. $\boldsymbol{A}$, Example traces from one experiment in awake and anesthesia showing the typical LFP signature following stimulus onset. $\boldsymbol{B}$, Box plot of the latency of the 


\section{continued}

stimulus onset to the trough and peak of the LFP signature $* 1.5 \mathrm{SD}$ of the mean $(n=17)$. C, Comparison of the time between trough and peak in each condition. $\boldsymbol{D}$, Comparison of amplitude $(\mathrm{mV})$ of troughs and peaks in each condition. $\boldsymbol{E}$, Top, Average stimulusevoked LFP in awake and anesthesia across all experiments ( $n=25$ in nine animals). Error bars indicate SEM. Bottom, Morlet wavelet of LFP activity following visual stimulation in the awake and anesthetized animals. $\boldsymbol{F}$, Stimulus evoked firing rates (top) and raster plots (bottom) of all trials in all units in each state. One line represents one trial ( 96 trials per unit). Trials are ranked according to awake firing rates from high to low rate from (bottom up) for each anesthetic. G, PSTH of evoked firing rates for all units followed between the awake and anesthetized state (error bars, SEM). $\boldsymbol{H}$, PSTH of normalized firing rates spanning the time period of the average peak of evoked activity. I, Scatterplot showing latency $(\mathrm{ms})$ to the first peak evoked response for all units in awake and anesthesia. Red dotted line indicates regression. $\boldsymbol{J}$, Box plot comparing bins of first peak max responses and first peak onset for all units between awake and anesthesia $(n=262,130)$. $K$, Frequency distribution of the awake-anesthesia difference in latency for each unit. $\boldsymbol{L}$, Box plot showing first peak latency for units in three anesthetic regimes (Iso/Dor $n=133$, Iso $n=71$, Ket/Xyl $n=47$ ).

each unit. Figure $2 F$ shows the spiking response of all units across all trials, as an average (top panel) and to each stimulus trial (bottom panel). To investigate the time frame of the elicited response, we computed PSTHs using a Gaussian smoothing kernel with 10-ms bins for each unit. In the awake state, the average PSTH for all units had a clear initial peak of activity with a shorter latency compared to anesthesia (Fig. $2 F, G$ ). To rule out that this pattern was not caused by the activity of a few dominating units, several tests were conducted. First, normalizing the firing rates to baseline levels showed the same tendency (Fig. $2 H$ ). Normalization was performed by quantifying unit firing rate $(R 1)$ of each bin to the mean firing rate of that unit (R2) (R1 - R2)/(R1 + R2). Next, we measured the time to first peak for each unit. The first peak following stimulus onset was included if the firing rate exceeded 1 SD of the mean firing rate of the unit. The time to the first peak after stimulus onset in the awake state was significantly slower during anesthesia (latency awake: $105 \pm 4 \mathrm{~ms}$, anesthesia: $153 \pm 5$ ms, $p<0.0001$; Wilcoxon, $n=172$; Fig. 2l,J). Following the same units from the awake to anesthetized state allowed a direct comparison of the response time in the two states (Fig. $2 /, \mathrm{J}$ ) and revealed that most units showed a slower response to stimuli in the anesthetized state. To assess whether the delayed response also was present for the onset of the stimulus-evoked peak, we estimated the onset as the time point where firing rate exceeded $10 \%$ of baseline activity and remained above this level for at least 25 ms (Brincat and Connor, 2006). We find a significant delay of stimulus-evoked peak onset activity between the awake and anesthesia condition for our units (latency awake: $75 \pm 6 \mathrm{~ms}$, anesthesia: $104 \pm 6$ ms, $p<0.0001$; Wilcoxon, $n=130$; Fig. $2 J)$. The mean delay between states for the first peak measure was 48 $\mathrm{ms}$, with the majority of units predominantly spanning 35-62 ms (95\% confidence intervals; Fig. 2K), while a mean delay of $30 \mathrm{~ms}$ was present for peak onset (spanning 14-44 ms, 95\% confidence intervals; Fig. 2K). Furthermore, although a weak but positive correlation between latencies in the awake anesthetized states was observed ( $r=0.16, p=0.0383, n=172$; Fig. $2 /$ ) a widespread distribution of responses across populations was present (slope $=0.14,95 \%$ confidence interval $=$ $-0.081-0.36$, y intercept $=138.5,95 \%$ confidence interval $=112.8-164.2)$ suggesting that latency under anesthesia was largely independent of latency in the awake state. A delay appeared to be present in all three anes- thetic regimes tested; but it was only significant for two regimes: Isoflurane and Isoflurane/Dormicum [Fig. 2L; Iso/ Dor aw-an $p<0.0001(n=90)$. Iso aw-an $p=0.024(n=$ 45). Ket/Xyl aw-an $p=0.10(n=37)$; Wilcoxon]. Furthermore, the Isoflurane/Dormicum condition had a larger delay than the two other conditions (Iso vs Iso/Dor $p<$ 0.005 , Iso/Dor-Ket/Xyl 0.0006, Iso vs Ket/Xyl n.s., Kruskal Wallis, Dunn's post hoc test).

\section{Pair-wise correlations increase under anesthesia}

Temporal structure of neuronal firing can be investigated by comparing correlations between spike-times of pairs of neurons. Although these correlations are often modest in magnitude, they may reflect strong constraints on information processing at the population level (Schneidman et al., 2006). It is still debated to what extent the activity of neurons in sensory cortices are correlated, with estimates ranging from $1 \%$ to $40 \%$ (Schulz and Carandini, 2010). Previous work shows that the activity of sensory neurons in layer $2 / 3$ is more correlated during anesthesia than in the awake state (Greenberg et al., 2008). We compared correlations between unit pairs during the awake state, anesthesia, and recovery across cortical layers. Correlations were computed on pairs from the same local region (position of the tetrode wire bundle).

Figure $3 A$ shows the correlation matrix within a population across the states. The pair-wise CC were substantially higher in the anesthetized state compared with the awake state [Fig. $3 B$; awake: $0.009 \pm 0.026(n=733)$ vs. anesthesia: $0.026 \pm 0.040(n=1053), p<0.0001$. Friedman, Dunn's post hoc]. A small but significantly positive correlation was present between pair-wise CC in the awake state and the same cells pair-wise CC in anesthesia (Fig. 3C, $r=0.26, p<0.0001, n=1053$, Spearman). This indicates a weak relationship between a cell-pair's correlated activity pattern in the awake state to that same cell-pair's activity in the anesthetized state (Fig. 3C, 7 outliers out of 1053 pairs were removed that exceeded 5 $\mathrm{SD}$ from the mean, for graphical presentation).

Interestingly, during the recovery sessions after anesthesia, the pair-wise CC were significantly lower $(p=$ 0.025 , Dunn's post hoc) than in the initial awake sessions (recovery: $0.006 \pm 0.023, n=733$ ). To examine the impact of recovery time, we separated the recovery sessions into those cases that recently recovered from the anesthetic but were apparently fully awake (recovery 1, 15-30 min after righting reflex is restored) and where the 
A
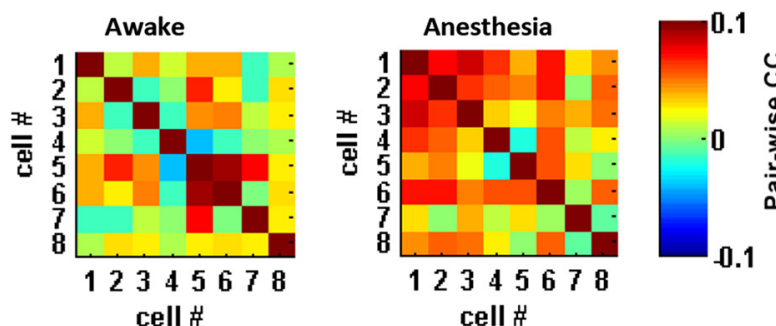

0.1

\section{B}

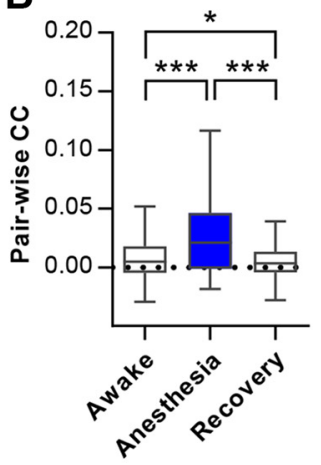

C

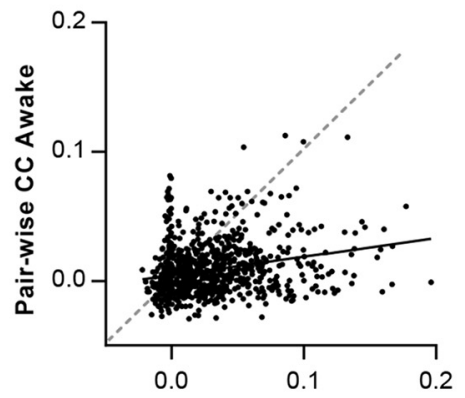

Pair-wise CC Anesthesia

E

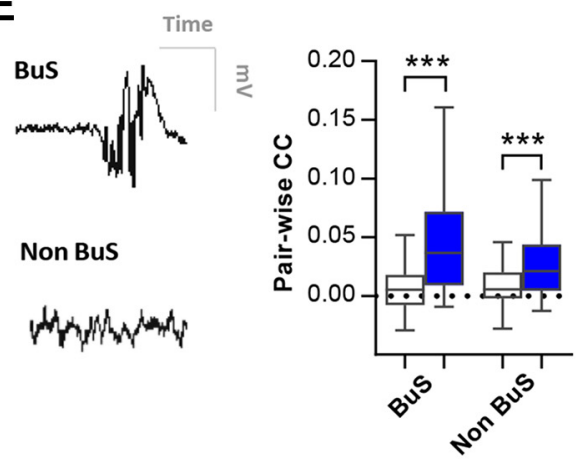

G

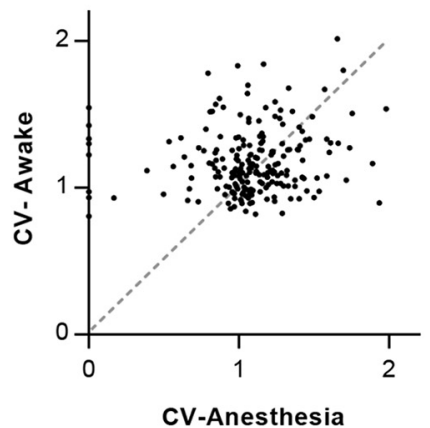

D

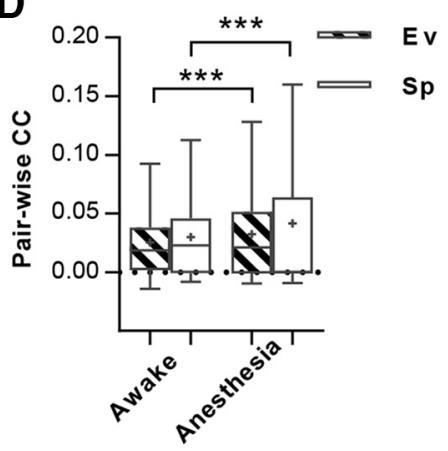

F

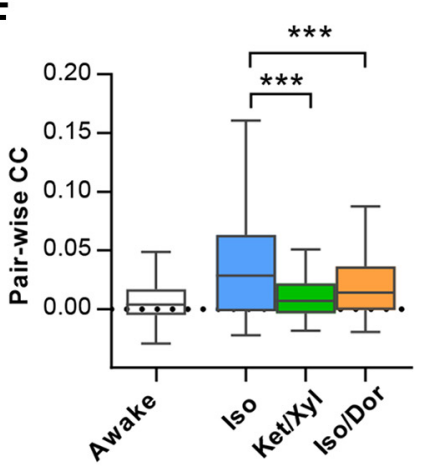

H

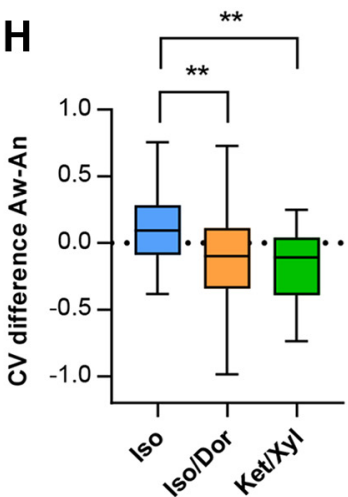

Figure 3. Temporal structure parameters, pairwise correlations and CV, of units followed between states. $\boldsymbol{A}$, Pair-wise CC matrix for an example population (8 units) in both states. B. Box plot showing the CC for all pairwise correlations in awake, anesthesia and recovery ( $n=733$ cell-pairs). C, Scatter plot of pair-wise CC for pairs of neurons in the awake and anesthetized state $(n=1046)$. Black line indicates regression. $\boldsymbol{D}$, Box plot showing the CCs during stimulus-evoked and spontaneous activity for all units. $\boldsymbol{E}$, left panel, Example LFP trace with a typical burst suppression (BuS) pattern and no burst suppression (non-BuS). Right panel, Box plot showing CCs for pairs in sessions dominated by burst suppression $(n=276)$ compared with non-BS sessions $(n=210)$. $\boldsymbol{F}$, Box plot showing the CCs for cell-pairs in three different anesthetic regimes: Isoflurane $(n=371)$, Ketamine/Xylazine $(n=246)$, and Isoflurane/ Dormicum $(n=436)$. G, Scatter plot of the CV for single units in the awake and anesthetized state $(n=218)$. $\boldsymbol{H}$, Box plot of the awake-anesthetized CV difference for units in each anesthetic regime (Iso $n=56$, Iso/Dor $n=118$, Ket/Xyl $n=44$ ). 
rats had recovered for $24 \mathrm{~h}$ (recovery 2). The correlations were significantly lower immediately after waking up from anesthesia compared with the preanesthetic awake condition ( $p=0.0004, n=651$, Friedman, Dunn's post hoc test). In contrast, after $24 \mathrm{~h}$ of recovery (recovery 2) correlations were similar to the awake state before anesthesia ( $p=0.635, n=393)$. This suggests a more pronounced desynchrony among cell pairs immediately following recovery from anesthesia, which is restored as the animal fully recovers from the anesthetic. This may have implications for experiments where the animal undergoes surgery on the day of recording.

Next, we investigated how pair-wise CC within stimulus-evoked and spontaneous time periods were affected by anesthesia. Our data show higher correlations in anesthesia compared to awake in both evoked and spontaneous time periods (Fig. 3D; evoked, $p<0.0001, n=$ 1039 , spontaneous, $p=0.042, n=962$; Wilcoxon). Also, during spontaneous time periods, we find higher correlations compared to stimulus presentation in both the awake condition and under anesthesia (awake: $p<$ 0.0001, $n=1049$; anesthesia: $p=0.04, n=959$ ). Thus, the activity seems to be more correlated when the screen is blank/gray than during stimulus presentation in our population of pseudo-randomly sampled units from all layers of the neocortical column. This is in contradiction to what was reported by Hofer et al. (2011) for layer 2/3.

Burst suppression and shorter periods with an isoelectric trace in the electroencephalogram are common during Isoflurane anesthesia and have the potential to increase the correlation estimate, since units timelock their firing to bursts (UP states) (Steriade et al., 1994). We therefore tested how burst suppression during anesthesia affects synchrony among cell pairs compared with sessions not dominated by burst suppresssion in the LFP (Fig. 3E). As expected, periods with burst suppression showed the highest pair-wise CC ( $p<0.0001, n=276$; Wilcoxon). However, sessions lacking burst suppression still showed higher pair-wise CC compared to the awake state $(p<$ $0.0001, n=210$; Wilcoxon), suggesting that burst suppression patterns are not the sole cause of increased pair-wise correlations during anesthesia. The two comparisons were also found to be significantly different from each other ( $p<0.0001$, Mann-Whitney), indicating that burst suppression causes a greater correlation in anesthesia compared with sessions with non-burst suppressed LFP. Comparing the effect of the three different anesthetic regimes, the Isoflurane condition showed significantly higher CCs (Fig. 3F; Iso vs Iso/Dor $p<0.0001$, Iso vs Ket/Xyl $p<0.0001$, Iso/Dor-Ket/Xyl n.s.; Iso/Dor $n=436$, Iso $n=371$, Ket/Xyl $n=246$; Kruskal-Wallis, Dunn's post hoc test). The difference is likely due to the high prevalence of burst suppression under Isoflurane anesthesia.

The coefficient of variation (CV) was used to quantify the degree of spiking variability in single units between states. We find a higher $\mathrm{CV}$ in the awake state compared with CV in across all anesthetics $(p=0.003, n=218$; Wilcoxon), indicative of more regularity of the firing of a unit during anesthesia (Fig. 3G; five outliers that exceeded
$3 \mathrm{SD}$ from the mean were excluded for the graphical presentation). However, we also find differences in the impact of different anesthetics. For both Ket/Xyl and Iso/ Dor, there is a significant decrease in CV with anesthesia (awake-Iso/Dor $p=0.002, n=118$, awake-Ket/Xyl $p=$ $0.0002, n=44$ ); however, for Isoflurane only, there is no significant increase in CV with anesthesia (awake-Iso: $p=$ $0.067, n=56$ ). When directly compared, the Isoflurane condition differs significantly from the other two anesthetics (Fig. $3 H$; Iso vs Iso/Dor $p=0.004$, Iso vs Ket/Xyl $p=$ 0.0004 , Iso/Dor vs Ket/Xyl n.s.; Kruskal Wallis, Dunn's post hoc test).

\section{Preservation of temporal sequences}

Our findings of increased evoked latencies in units and LFP and changes in pair-wise correlations in response to anesthesia suggest a different temporal response profile in anesthesia compared to the awake state. In light of previous findings of preserved population-based sequences in auditory and somatosensory cortex during awake and anesthesia (Luczak et al., 2007, 2009), we examined if such temporal sequences also exist in local ensembles of the visual cortex and whether such sequences are preserved between the awake and anesthetized states.

We based our analysis on the method described by Luczak et al. (2009) with modifications to account for the reduction in firing rates, differences in evoked latencies between the states and smaller population sizes.

First, we compared the sequence of mean spike latencies (MSLs) in the populations across and within states. MSL was quantified as the mean spike time of each unit during a time span of $200-m s$ poststimulus onset. We used this 200-ms time window since most units respond maximally during this time period, in both the awake and anesthetized condition (Fig. $2 /, J$ ). To visualize a potential sequential activation within and between states, we sorted the MSLs of one session and plotted the corresponding unit MSL in the other session. Figure $4 A$ shows the MSL (red dots) and firing rates normalized to maximum and minimum in gray pseudocolor of all units, with a minimum of 9 units in each population. The preservation of sequential firing structure can be observed in all state comparisons, although the correlation is weaker comparing the awake and anesthesia sessions (awake-awake (awaw): $r=0.46, p<0.0001, n=107$, anesthesiaanesthesia (anan): $r=0.42, p<0.0001, n=91$, awakeanesthesia (awan): $r=0.30, p=0.0031, n=93$; Spearman). To further investigate if the sequential firing structure with the MSL measure was present with individual ensembles, we analyzed individual population MSLs with a minimum of 15 units followed between states. In these ensembles we found mostly significant correlations for the awake-awake and anesthesia-anesthesia comparisons [awake-awake: mean $r=0.57, p<0.05$, four populations (one population n.s.), Spearman, $n=15,19,18,18$, anesthesia-anesthesia: mean $r=0.66, p<0.005$, three populations, $n=17,17,18]$. In contrast, only one of four populations showed preserved sequence between awake 
A
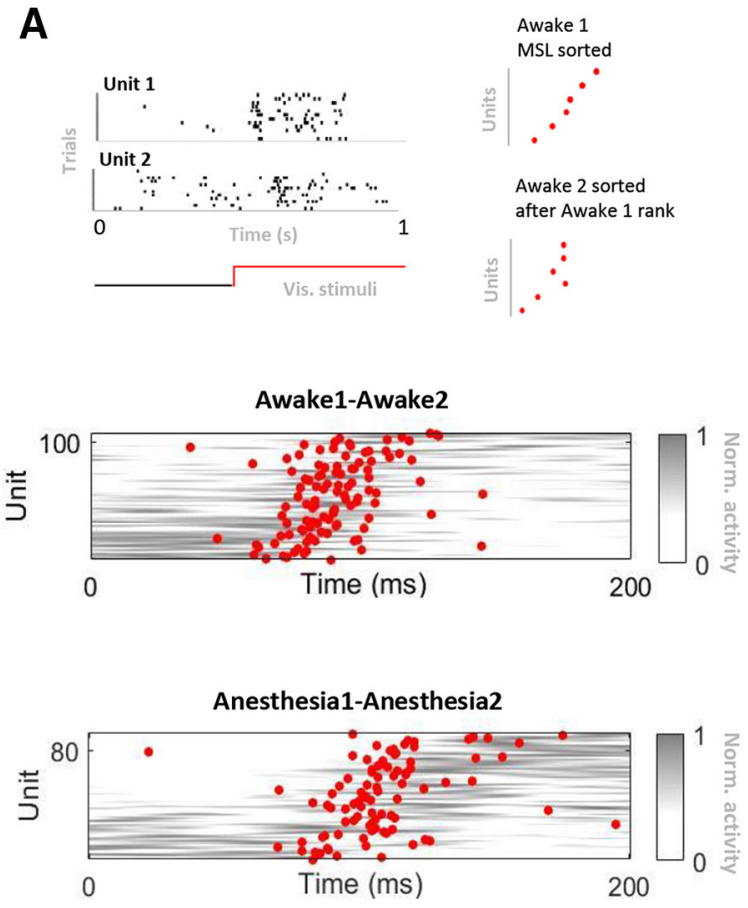

Awake-Anesthesia

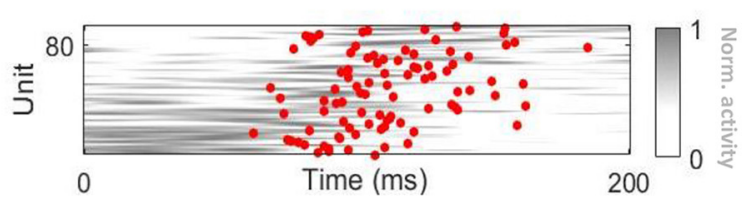

B

Single rank trial
correlation

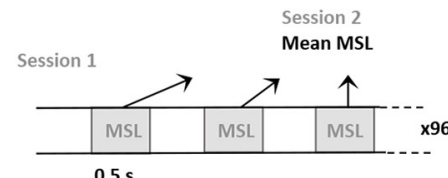

Visual stimulus
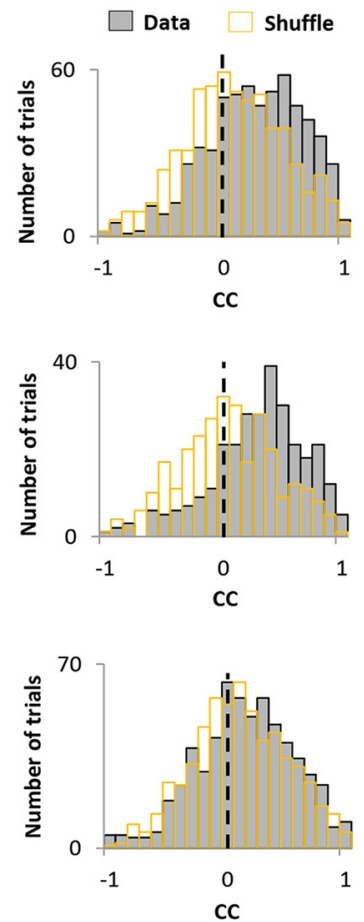

C

Rank by rank

measure

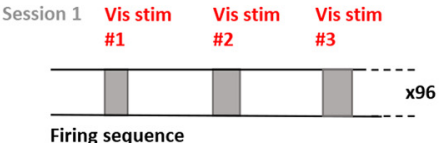

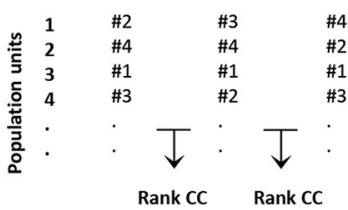

x96 $=$ Average Rank CC session 1

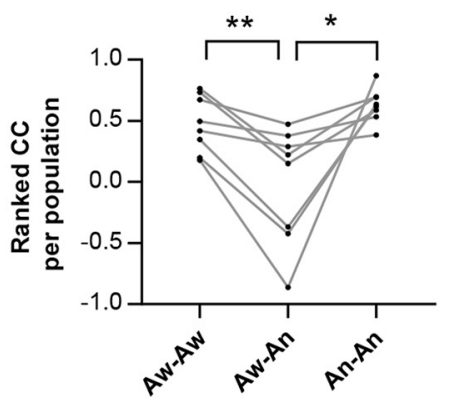

D
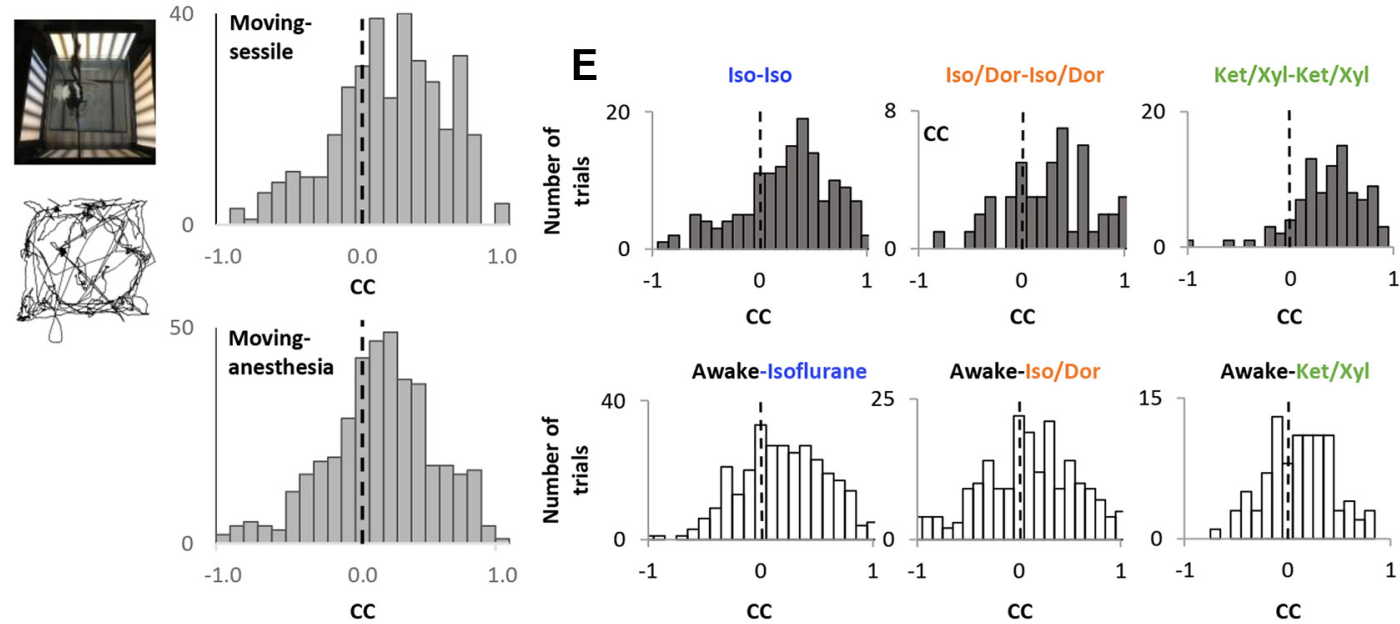

Awake-Iso/Dor

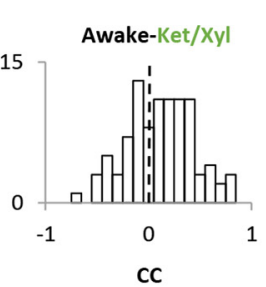

F

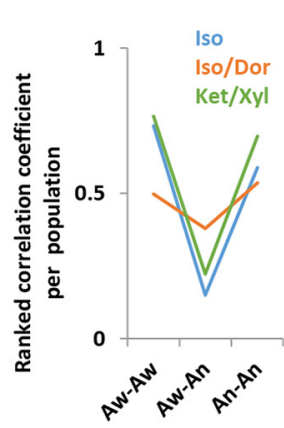

Figure 4. Temporal sequences within unit ensembles followed between states. A, Top panel, Description of MSL measure. Left, Raster plot of two representative units firing with different MSL to visual stimuli. Right, illustration of sequence representation for MSL measure. Bottom panels, MSL (red dots) for units within ensembles, sorted by their ranked sequence (MSL) in the other session. Gray indicates activity normalized between 0 and 1 (awake-awake, $n=88$; awake-anesthesia, $n=82$; anesthesia-anesthesia, $n=78$ ). $\boldsymbol{B}$, top panel, Illustration of the quantification of the single trial rank measure. Bottom panels, Histograms of single trial rank CCs. MSLs for individual stimuli presentations are rank correlated with the mean latency response across many stimuli presentations from a separate experimental session (awake-awake, $n=604$; awake-anesthesia, $n=596$; anesthesia-anesthesia, $n=294$ ). Yellow outline indicates shuffled data. $\mathbf{C}$, top panel, The rank-by-rank measure is described. Bottom panel, Line graph showing the CCs from the rank-by-rank measure for the individual populations $(n=8$ populations). $\boldsymbol{D}$, Single trial rank correlations for the sessile-movement $(n=$ 
continued

352) and movement-anesthesia $(n=402)$ comparisons. $\boldsymbol{E}$, Single trial rank correlations for the awake-anesthesia and anesthesiaanesthesia comparisons for three different anesthetic regimes: Isoflurane, isoflurane-Dormicum, and Ketamine/Xylazine. $\boldsymbol{F}$, Example populations, from the three anesthetic regimes (Iso $n=15$ units, Ket/Xyl $n=19$, Iso-Dor $n=8$ ).

and anesthesia [mean $r=0.37,4$ populations (n.s. except 1 population) $n=15,17,17,18]$.

Second, we looked at trial correlations between the MSL for the units following a single visual stimuli presentation and the average MSL of the unit from the other state. This was performed to investigate whether ensemble responses for individual visual stimuli presentations correspond to the averages assessed in the previous MSL measure (Fig. 4A). For this assessment, all single rank correlations are shown that include a minimum of six units firing in response to a stimulus (Fig. 4B). The histograms of Figure $4 B$ shows the distribution of the rank correlations in each state comparison (96 visual stimuli repetitions per averaged MSL measure). The positive skew in all single rank correlations illustrates that for the majority of stimulus presentations the firing sequence was preserved, particularly when comparing awake to awake and anesthesia to anesthesia. Furthermore, the awake and anesthesia comparisons also reveal a slightly skewed distribution of single rank correlations indicating some preservation of stimulus induced sequences across states. To assess the skewness of the distributions a $t$ test to a theoretical mean of zero was performed (awaw $p<$ 0.0001 , awan, $p<0.0001$, anan $p<0.0001, n=288$; $t$ test). Importantly, although we find a positive skew in all state comparisons, we also find that the correlations within states are significantly different from correlations between states, i.e., the sequence preservation between awake-anesthesia is significantly less than what is present between awake-awake and anesthesia-anesthesia [p < 0.0001 , Kruskal-Wallis; awake-awake vs awake-anesthesia (awaw-awan): $p<0.0001$, awake-anesthesia vs anesthesiaanesthesia (awan-anan): $p<0.0001$; Dunn's post hoc; awake-awake: $n=604$, awake-anesthesia: $n=596$, anesthesia-anesthesia: $n=294]$.

To verify that the sequence of unit- firing was not due to systematic changes in firing rates between the states, we conducted a random shuffling of unit identity. To maintain all firing rate distributions in response to a stimulus, the random assignment of cell identity was considered to be the most robust test, i.e., shuffling unit position within a population. Figure $4 B$ shows the shuffling results (yellow region) for the single trial rank. As expected, for all measures the shuffling resulted in no skewness and normal distribution of events (awaw $p=0.347$, awan, $p=0.781$, anan $p=0.411, n=288$; $t$ test to a theoretical mean of 0 ), and no differences between the comparisons $(p=0.427$, $n=288$, one-way ANOVA).

When analyzing single rank trial correlations from awake-anesthesia to shuffled awake-anesthesia we find that the correlations (the positive skew) between awake and anesthesia is significantly different from random shuffling of unit positions ( $p=0.011, n=596)$. This illustrates that although the sequence preservation between awake and anesthesia is weakened, there is still some preserva- tion intact. However, the preservation observed in awakeawake and anesthesia-anesthesia comparisons to the shuffle data are far stronger $(p<0.0001)$, suggesting that the majority of temporal sequence preservation is state dependent and show reduction between states.

Third, we implemented a new measure to reduce the impact of firing rate and mean latency on the overall scores. The rank-by-rank measure individually ranks the firing sequence of a population for each stimulus (96 repetitions) and creates an average rank score for each experimental session. This average rank score is then correlated to a corresponding measure from a separate experimental session for the same population. This way, only the relative rank position of the firing of the unit in a response to a stimulus is kept and averaged. Using this analysis we confirm that the transition between awake and anesthesia impairs the sequence preservation observed within states, i.e., CCs are significantly higher in awake-awake comparisons and anesthesia-anesthesia comparisons (Fig. 4C; ANOVA, $p=0.01$; Tukey's, awawawan: $p<0.01$, awaw-anan: $p=0.36$, awan-anan: $p<$ $0.05, n=8$ populations). Shuffling unit identity within the ensembles removed all differences. More populations are included in this measure versus the single populationMSL correlations (first measure) since the rank-by-rank measure averages ranks across several repetitions and thus yields a more robust number across stimulus presentations, less units per ensemble is therefore required to produce meaningful data on activity within the ensemble. For populations to be included in the rank-by-rank measure more than six units in an ensemble had to be active (vs 15 units for MSL comparisons). The CCs from the rank-by-rank measure for the separate populations (Fig. 4C) shows that for all populations there is a lower correlation in the awake-anesthesia comparison.

Further, to show that our results were not derived from a potential variation in spatial frequency perceived by the animal between the sessile awake and anesthetized state, we quantified single stimulus rank correlations in movement sessions. During movement sessions the rat freely roamed the recording box and thereby sampling of the maximum and miniumum distance to each screen was covered throughout the experimental session. We find reduced sequence preservation in the ensembles between the wakeful moving and anesthetized state, compared with the stronger preservation between the moving and sessile session (Fig. 4D; sessile-moving vs movinganesthesia: $p=0.0131, n=352 / 402$, Mann-Whitney, five ensembles).

Finally, to ensure that our results were not derived from the use of a single anesthetic, we tested three different anesthetic regimes. We find similar results in each regime. Similarly, the single stimulus rank correlation (Fig. 4E) shows a positive skew for all anesthesia-anesthesia comparisons, while awake-anesthesia has a less positive 
skew. Shuffling the data again removes this positive skew. Figure $4 F$ shows an example population from each anesthetic regime showing the reduction in temporal sequence preservation for the rank-by-rank measure between awake and anesthesia compared with awake-awake and anesthesia-anesthesia.

\section{Responses between states are more similar at lower spatial frequencies}

Previous studies report unit preferences to drifting gratings of particular spatial and temporal frequencies in anesthesia (Girman et al., 1999; Niell and Stryker, 2008). To date, no reports have investigated how such preferences may change for individual units from the awake to the anesthetized state. In light of the findings of changes in spike timing to visual stimulation in anesthesia, we hypothesized that an altered temporal visual processing demand could affect the unit firing responses. Changing the spatial and temporal frequencies of the stimulus were considered to represent different visual processing demands, indicating the effect of spike timing changes on visual information processing.

We first investigated spatial frequency responses and presented sinusoidal drifting gratings with a fixed temporal frequency ( $\sim 4 \mathrm{~Hz}$ reported preference for rats; Girman et al., 1999) and varied the spatial frequency. In the awake state, the animal was allowed to move in a transparent box $(28 \times 28 \mathrm{~cm})$ surrounded by monitors presenting the visual stimulation. To calculate the maximum variation in spatial frequency introduced by the rat's range of locations in the box, each grating cycle size was estimated at the location furthest and closest from the screen to the rat's eyes (Fig. 5A). This showed that any spatial frequency response could maximally be skewed one spatial frequency group as a result of different locations of the rat in the chamber. The analyses were restricted to periods when the animals were sessile and the measurement of anesthetic responses was conducted with Isoflurane/Dormicum anesthesia due to the low changes in firing rate over time with this anesthetic (Fig. 1K).

Comparing the same units across states we found a general tendency across units to prefer lower spatial frequencies in anesthesia compared to the awake state (Fig. $5 B, C)$. Specifically, preferred frequencies during anesthesia were between $0.02-0.08 \mathrm{c} / \mathrm{d}$, like previously reported (Girman et al., 1999; Niell and Stryker, 2008), while the normalized responses to the highest spatial frequency $(0.3 \mathrm{c} / \mathrm{d})$ was significantly lower in anesthesia compared to awake (Fig. 5B, $p=0.012, n=68$; Wilcoxon). Furthermore, the units differed in their change of preferred spatial frequencies, where 28 units shifted toward lower, 22 units toward higher and 18 units showed no change in preferred spatial frequency. By separating and examining the units by their preferred spatial frequency in the awake state (Fig. 5D), we observed that units originally selective for the highest frequencies (right panel: 0.16 and $0.3 \mathrm{c} / \mathrm{d}$ ) in the awake state appeared to shift their preferences toward lower (left panel: 0.02-0.04 c/d) frequencies during anesthesia. In contrast, units originally selective for the lower frequencies, showed no systematic change with anesthesia. This was true both for normalized activity during the different stimulus presentations and the units' preferences. Interestingly, no shift was present in the units preferring $0.08 \mathrm{c} / \mathrm{d}$. There was a significant correlation for the normalized activity of low frequency selective units between awake and anesthesia $(r=0.44, p<0.0001$, Spearman, $n=75$; Fig. $5 D$, left panel). For the high frequency selective units we found no correlation between the preference in the two states indicating a change of preference between states toward the lower end of the spectrum ( $r=-0.08$, n.s, Spearman, $n=75$; Fig. $5 D$, right panel).

We also investigated the first peak latency of units during the different spatial and temporal frequencies (Fig. $5 G, J$ ). Interestingly, in anesthesia there appeared to be a gradually decreasing latency with each increase in spatial frequency (Fig. 5E-G) while the latencies were surprisingly similar in the awake state in all spatial frequencies tested. Figure $5 E$ shows an example unit with a larger latency difference between awake and anesthesia in the spatial frequency $0.02 \mathrm{c} / \mathrm{d}$ compared with $0.3 \mathrm{c} / \mathrm{d}$. Units had an overall larger latency difference between awake and anesthesia during the lowest spatial frequency $(0.02 \mathrm{c} / \mathrm{d})$ compared with the highest $(0.3 \mathrm{c} / \mathrm{d}$; Fig. $5 G, p=0.03$, paired $t$ test, $n=39)$. The same was true for the second lowest $(0.04 \mathrm{c} / \mathrm{d})$ versus the highest $(0.3 \mathrm{c} / \mathrm{d} ; p=0.04$, paired $t$ test, $n=41$ ). Also, the majority of units in the 0.02 versus $0.3 \mathrm{c} / \mathrm{d}$ comparison showed this change in latency (Fig. 5F). There was still a significant first peak delay between awake and anesthesia in all conditions $(0.02 \mathrm{c} / \mathrm{d}$ : $p<0.0001, n=50 ; 0.04 \mathrm{c} / \mathrm{d}: p<0.0001, n=55 ; 0.08$ $\mathrm{c} / \mathrm{d}: p<0.0001, n=48 ; 0.16 \mathrm{c} / \mathrm{d}: p<0.0005, n=47 ; 0.3$ $\mathrm{c} / \mathrm{d}: p=0.019, n=41 ; 2 \mathrm{~Hz}: p=0.0003, n=48 ; 4 \mathrm{~Hz}:$ $p<0.0001, n=48 ; 8 \mathrm{~Hz}: p<0.0001, n=45$; paired $t$ tests). When testing the same units for visual stimuli with three temporal frequencies $(2,4$, and $8 \mathrm{~Hz})$, no significant differences between normalized firing rates and unit preferences was found between the awake and anesthetized state (Fig. $5 H, I)$.

In addition, no latency differences were found between the three different temporal frequencies in either state (Fig. 5J), suggesting that the varying effect of anesthesia on latency with no awake change is specific to activity under differing spatial frequencies. It would appear as the processing of spatial content rather than the contents speed is affected by anesthesia. Regardless, the presence of a state-dependent latency difference, which is determined by the spatial frequency of the visual stimuli highly suggests an altered visual processing during anesthesia.

\section{Modeling awake-anesthesia data}

Anesthetics appears to work by the direct effects on the neurons and indirect network effects due to the altered activity of neurons. In particular, several anesthetic agents seem to increase inhibition in a network. In principle, it should therefore be possible to account for the presently observed differences between anesthetized and awake brain states in a network model for visual cortex including sufficient biophysical details to mimic the effect of pres- 


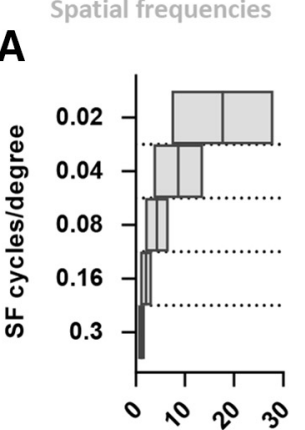

Size of cycle $(\mathrm{cm})$
B

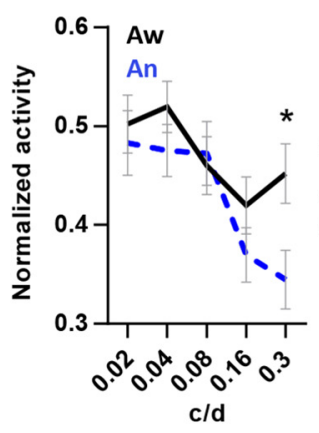

C

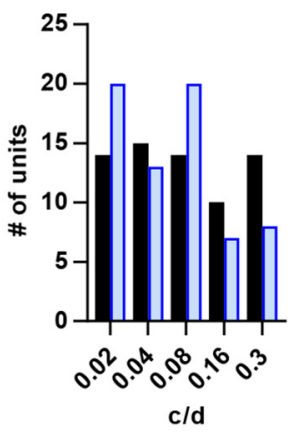

D

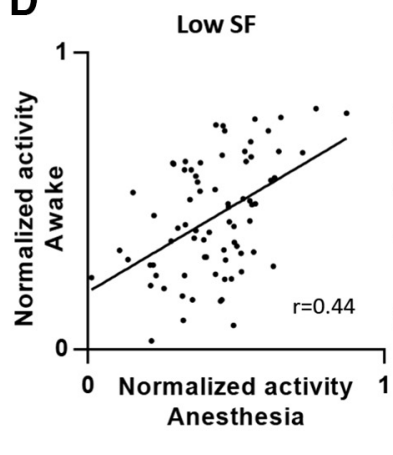

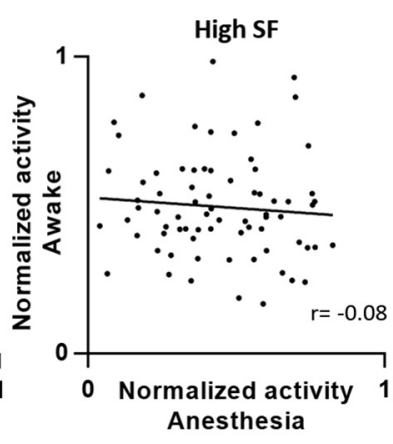

E
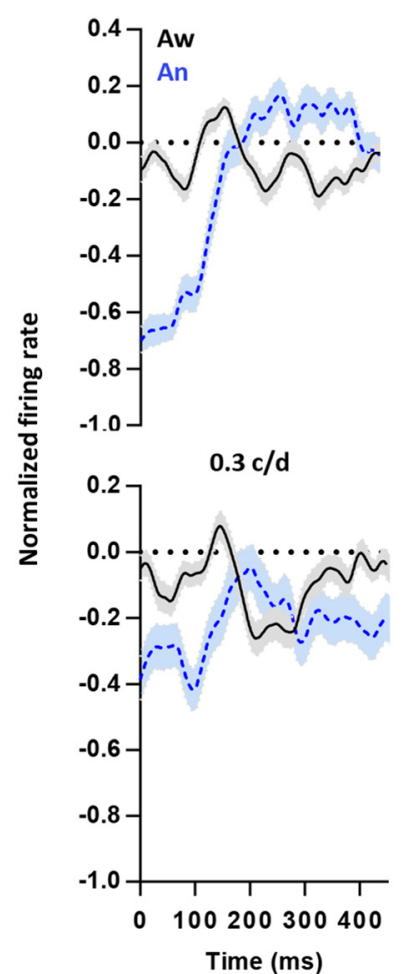

F

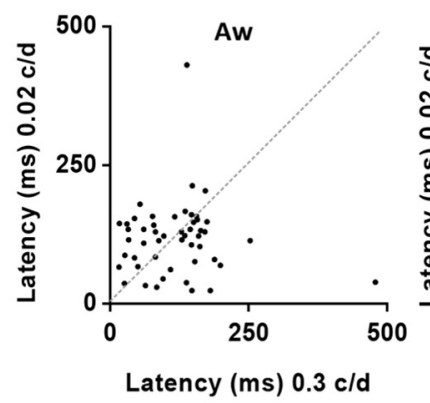

G

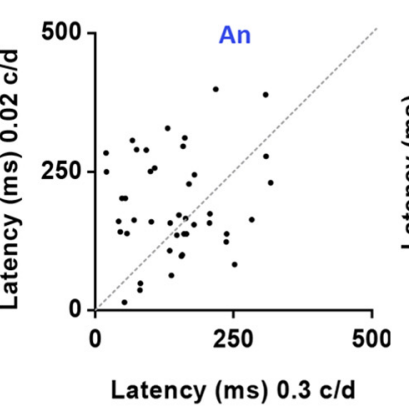

Latency $(\mathrm{ms}) 0.3 \mathrm{c} / \mathrm{d}$
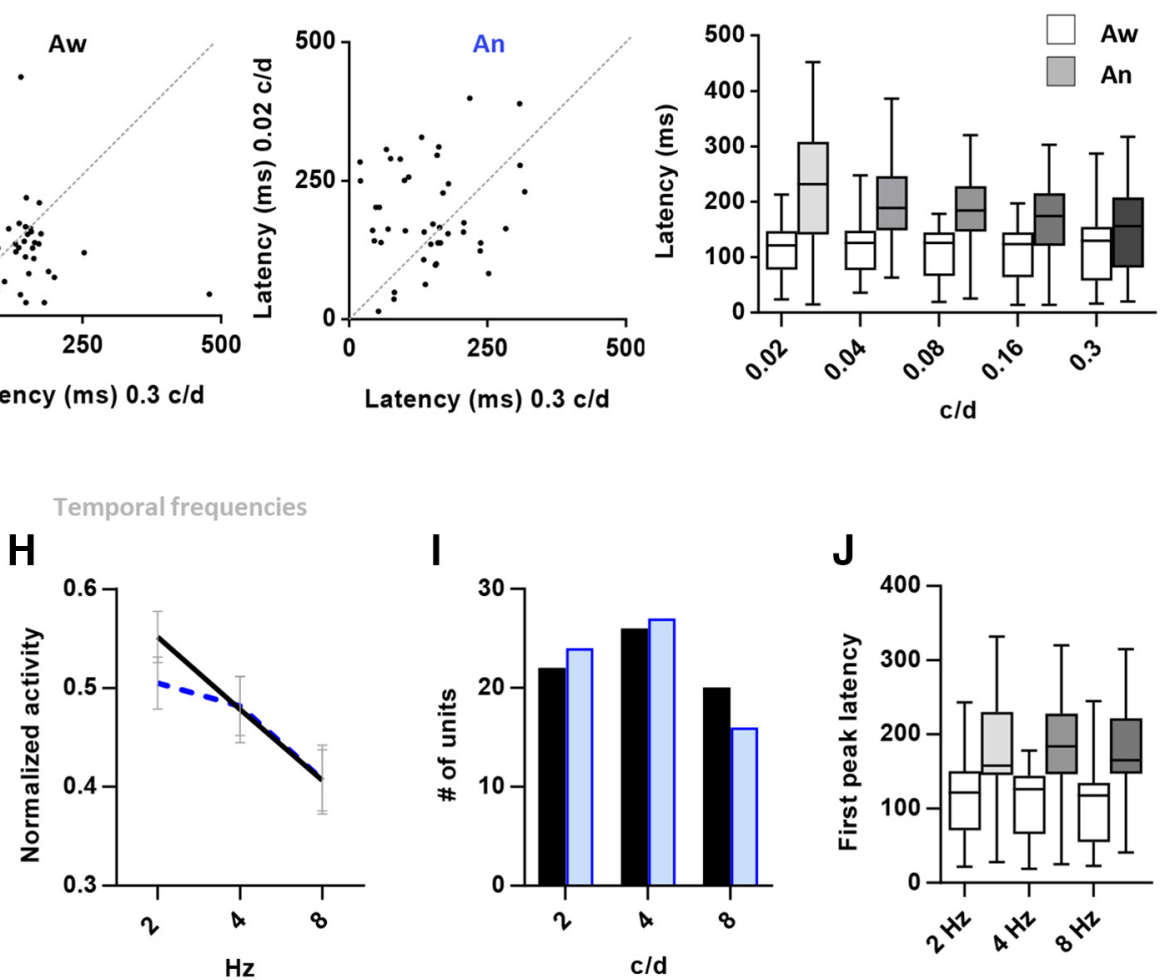

Figure 5. Responses to visual stimuli of different spatial frequencies between awake and anesthesia (A-G). $\boldsymbol{A}$, The possible position of the awake rats in the recording box gives a theoretical range of spatial frequencies of the stimuli. The maximum and minimum size of cycles considering varied distance to screen is plotted and show that overlap is restricted to one spatial frequency group. $\boldsymbol{B}$, Average normalized firing rates for all units in each spatial frequency $(n=68)$. Error bars indicate SEM. Firing rates normalized by scaling between 0 and 1. C, Number of units responding maximally to each spatial frequency in awake and anesthesia. $\boldsymbol{D}$, left panel, Comparison of normalized activity in awake and anesthesia during visual stimuli with low spatial frequencies (0.02 and $0.04 \mathrm{c} / \mathrm{d})$ for units preferring a low spatial frequency stimuli in the awake state $(n=75)$. Right panel, Same for units preferring high spatial frequencies of visual stimuli in awake $(0.16$ and $0.3 \mathrm{c} / \mathrm{d} ; n=75)$. $E$, PSTH for an example unit during visual stimuli with a low spatial frequency (top panel) and a high spatial frequency (bottom panel) in the awake state and during anesthesia. $\boldsymbol{F}$, Scatterplot of evoked latencies for the lowest spatial frequency $(0.02 \mathrm{c} / \mathrm{d})$ and the highest spatial frequency $(0.3 \mathrm{c} / \mathrm{d})$ for the awake state (left panel) and during anesthesia (right panel). G, Box plot of first peak latencies for units under five different spatial frequencies during awake and anesthesia. Responses to visual stimuli of different temporal frequencies between awake and anesthesia $(\boldsymbol{H}-\boldsymbol{J})$. $\boldsymbol{H}$, Average normalized firing rates for all units in each temporal frequency. $I$, Number of units responding maximally to each temporal frequency in awake and anesthesia. $\boldsymbol{J}$, Box plot of first peak latencies for units under visual stimulation with three different temporal frequencies during awake and anesthesia. Error bars indicate SEM. Firing rates normalized by scaling between 0 and 1.

ence or absence of the anesthetic agent. The present type of data where the neural activity is measured for the same set of cells in both the anesthetized and awake states allows for a new set of validation tests that candidate cortical network models should pass. To illustrate this approach we next compare our experimental results with predictions from model simulations using NEST (nestsimulator.org; Gewaltig and Diesmann, 2007). Here, we 
consider one of the most well-established and analyzed network models mimicking cortical network activity, namely the Brunel network (Brunel, 2000) with two recurrently connected populations, one excitatory and one inhibitory, of LIFs.

As described in detail in Materials and Methods, the LIF neurons integrate synaptic input currents, and when the membrane potential reaches a preset threshold value, an action potential is added, and the membrane potential is reset to a preset reset value. Here, the neuron and network parameters were first set to give a network behavior plausibly mimicking an "awake" state, i.e., a state with asynchronous firing and firing rates in rough agreement with the present awake-state recordings. To mimic the anesthetized state of the same model network we modified the network in two ways. First the synaptic inhibition, i.e., the weight of the inhibitory synaptic current, was increased, and second the equilibrium value defined by the leak potential was lowered for all neurons in the network. The latter modification was meant to mimic an overall hyperpolarization of the neurons induced by a more global inhibitory action induced by the anesthetic agent. Most work on the Brunel model has considered homogeneous networks, i.e., the same synaptic weights between all neurons in the same population (Brunel, 2000). However, recently variants of the model including variations in the synaptic weights have been considered, and in particular a variant with lognormally distributed weights have been observed to make the dynamics more similar to experiments (lyer et al., 2013; Teramae and Fukai, 2014; Hagen et al., 2016). Here, we considered both homogeneous and "lognormal" networks to explore how robust our model findings were to this aspect of the network connectivity.

The simulation results were compared to the electrophysiological recordings in the two states and with different anesthetics, results are presented in Figure 6. Isoflurane-based anesthetics and the simulated data showed reduced firing in the anesthetized state, both the spontaneous rate (Fig. $6 A$, left panel) and the evoked peak rate (Fig. $6 A$, right panel). Both the homogeneous and the lognormal network showed the same trend, i.e., reduced firing rates in the anesthetized state, as the experiments for Isoflurane-based anesthetics. All three types of anesthesia were observed to increase the latency from stimulus onset to the peak response in the PSTH. This phenomenon was not captured in any of the models which both showed little variation of latency between the states (Fig. 6C). Note, however, that any increased latency at the geniculate level would not be captured in these models as the input to the network (mimicking input from LGN) was assumed identical in the awake and anesthetized states. However, both models captured the observed increase in pairwise CC (Fig. 6B, left panel). Anesthesia caused decrease in the $\mathrm{CV}$ in both models and the experiments, except for pure isoflurane where no change was observed (Fig. $6 B$, right panel).

Extracellular recordings cannot measure subthreshold variations of the membrane potential, but these are fully available in the models. As the models seemed to capture salient features of the differences between the awake and anesthetized states, we next investigated the models' distributions of subthreshold membrane potentials in the different states (Fig. 6D). These panels show the probability density of the membrane potentials where the population threshold distance is measured as percentage of the threshold value. The key observation was that in both models the distribution is narrower and more shifted toward the firing threshold in the anesthetized state than in the awake state (Fig. 6D). Despite this shift of the mean of membrane-potential distribution toward threshold with anesthesia (Fig. 6E, left panel), the firing in the awake states was still larger. This likely reflected the larger variance (Fig. 6E, right panel) in the awake state, i.e., that in the model the firing was more "fluctuation driven" than "mean driven" (Renart et al., 2007; Kriener et al., 2014).

Note that we consider these model observations to be quite preliminary and maybe primarily suited to illustrate a workflow for comparing experimental data with candidate network models. A more comprehensive comparison between models and the present experiments should be considered when more detailed candidate network models of the rat visual cortex becomes available.

\section{Discussion}

Visual cortical processing has been studied in great detail in anesthetized and awake head-restrained animals, but it still remains unclear how the temporal dynamics of neurons within an ensemble differ between states. Using chronic extracellular recordings of single units we show that simultaneously recorded units preserve a temporal sequence in response to visual stimulation. While this sequence of firing is maintained within states, it is weaker for the same ensemble across states indicating a change in temporal dynamics under anesthesia. This change is further confirmed from the slower stimulus-evoked responses during anesthesia compared to awake both on the population level (LFP) and in single unit responses and from the increase of pair-wise correlations between cell pairs. Moreover, we show that changing the spatial frequency of the visual stimuli results in a change in unit response-speed in anesthesia, while the same units remain more unaffected in the awake state. Comparing single-unit results such as these to network models is beneficial for improving models on network activity. We exemplify such a workflow by implementing a Brunel-type network model, and reproduce many features from the dataset in our model.

The findings that receptive field sizes are reduced in somatosensory cortex under anesthesia (ArmstrongJames and George, 1988) while they appear to increase in the visual cortex under anesthesia (Haider et al., 2013) highlights the need for separate investigations in each cortical area to reveal the impact of anesthesia on single units. This may in part be due to, e.g., differences in compositions of neuronal receptor subtypes that would make them differentially susceptible to a particular anesthetic drug, e.g., GABA-A receptor subunit combinations vary greatly between cortical areas, and several anesthetics are known to operate on different GABA-A receptor 
A

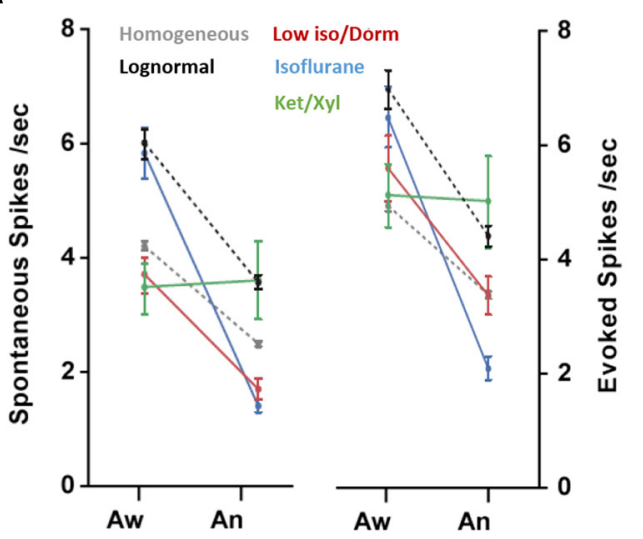

B

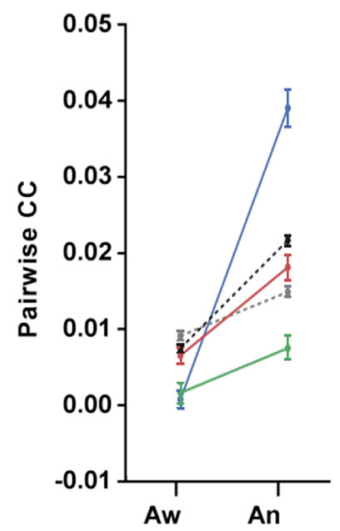

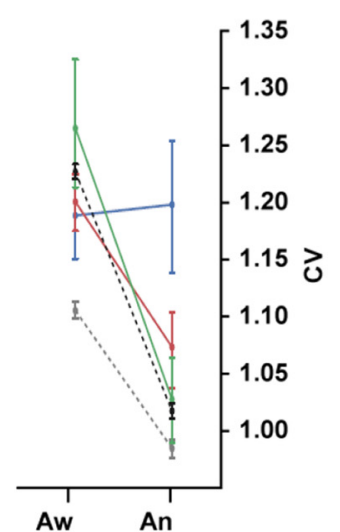

E

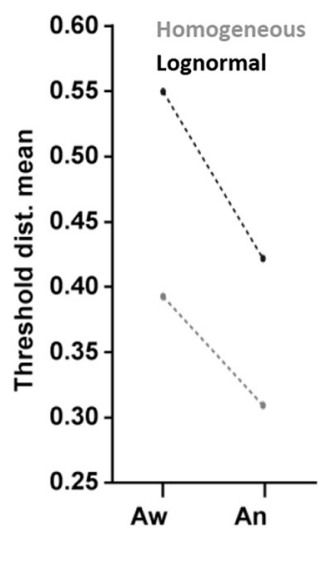

C

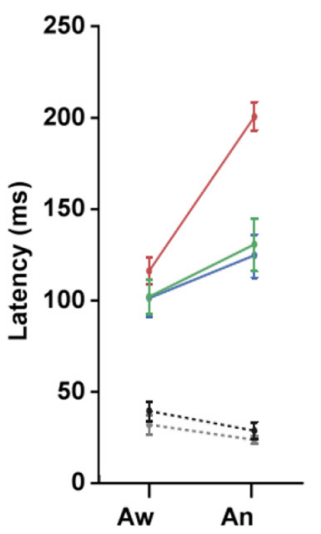

D

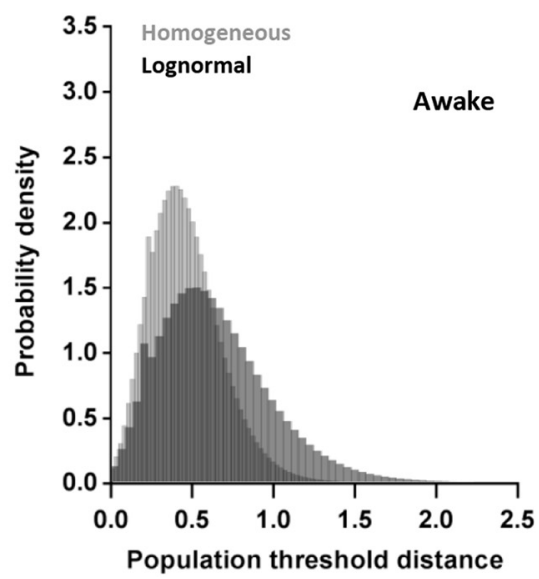

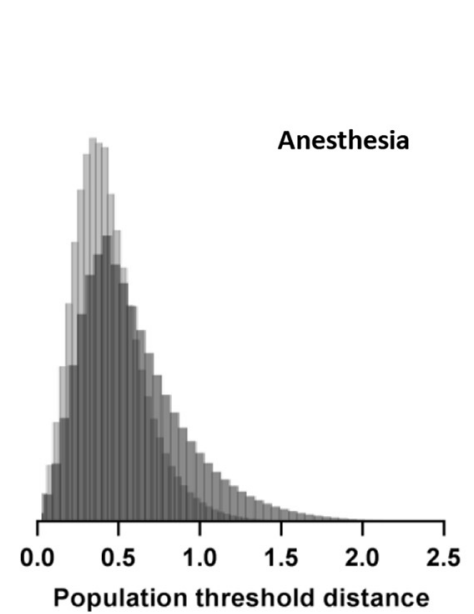

Figure 6. Awake-anesthesia data compared to data from the Brunel-type network model. Anesthetics are color coded. $\boldsymbol{A}$, left panel, Spontaneous rate. Right panel, Evoked rate. B, left panel, Pairwise CCs. Right panel, CV. C, Peak latency (ms). $\boldsymbol{D}$, Probability density of the membrane potential in the awake (left panel) and anesthetized (right panel) network model. $\boldsymbol{E}$, left panel, Network model threshold distributed mean. Right panel, Threshold distributed variance.

subunits. Thus the effects of anesthesia is likely agent and area specific (Vahle-Hinz and Detsch, 2002). For this reason, we compare three different anesthetic agents to reveal the effects of anesthesia on unit activity in the visual cortex.

\section{Chronic recordings}

Chronically implanted tetrodes may have several advantages compared to acute recordings. First, activity from the same units and LFP oscillations can be reliably followed across days enabling direct comparisons of changes to the neural population in response to manipulations. Second, recordings can be made in freely behaving animals lowering potential stress of restraint and potential impact of reduced head motility on population responses. Also, acute inflammatory responses due to electrode implantation is reduced as the animal is left to recover for several days after surgery after which the tetrodes are slowly lowered into the cortical column without reopening the implantation site. Although investigations of visually evoked processing in freely moving animals as they were conducted here, have their limitations with less control of eye movements and pupillary changes, our results show that reliable visual response patterns can be detected and that the cross-state recordings reveal properties of cell ensembles that are otherwise not possible to achieve.

\section{Diverse effects of anesthesia on single unit firing rates}

The strong reduction in firing rates in response to anesthesia (Fig. 1) is in accordance with previous findings from the visual cortex (Villeneuve and Casanova, 2003; Schummers et al., 2008; Sleigh et al., 2009; Vizuete et al., 2012). However, following the same population of units across states revealed nonhomogenous, cell-specific and individual unit differences in response to anesthesia (Fig. $1 C$ ), even among neighboring neurons (Fig. 1D). Since each neuron possess a different composition of receptor subtypes, display variation in neuronal morphology and electrophysiological response characteristics (Edwards et al., 1990; Steriade, 2004; Van Aerde and Feldmeyer, 2015) the variations of the effect of anesthesia could arise from local factors alone. In addition, each neuron also holds a unique position in the cortical circuitry, and as such the variation observed in reponses between neigh- 
boring neurons may be due to indirect network effects. Similarly, the larger reduction in firing rates of narrow spiking (putative interneurons) units compared to the broad spiking (putative excitatory) population (Fig. 1G) may reflect the molecular targets of anesthesia in the local circuitry (e.g., higher GABA receptor density on parvalbumin positive inhibitory neurons; Klausberger et al., 2002) or originate in altered upstream or presynaptic activity. Regardless, the different impact would produce highly different levels of inhibition between states (Haider et al., 2013). In contrast to the our findings, some previous reports find little impact on overall V1 firing rates with anesthesia (Pisauro et al., 2013; Durand et al., 2016). This could be partly explained by the use of urethane anesthesia in these experiments, since urethane has been shown to exert differential effect on neocortical neuronal activity compared to other anesthetics (Maggi and Meli, 1986; Dyer and Rigdon, 1987).

The increased evoked-spontaneous index in anesthesia compared to awake was mostly due to reduced spontaneous activity (Fig. 1L). This is consistent with findings from mice comparing separate populations (Niell and Stryker, 2010). The heavy reduction in spontaneous activity may be caused by reduced top-down and feedback processing in corticocortical connections during anesthesia (Mashour, 2014; Raz et al., 2014).

\section{Increased evoked latency during anesthesia}

The shorter stimulus-evoked latencies in the awake state compared to anesthesia in the LFP signature was reflected in the response latencies of single units (Fig. $2 B, I, \mathcal{C}$ ). The slower response to visual stimuli under anesthesia has implications for visual processing and interpretation of results from studies conducted under anesthesia. Following the units across states, we found that the slower response due to anesthesia differed between units giving an unpredictable effect on network processing. Numerous reports suggest that high temporal specificity is a trait of efficient processing, and that the coding of different signals is derived from slight variations in the temporal structure (Mainen and Sejnowski, 1995; Gasparini and Magee, 2006; Tiesinga et al., 2008; Luczak et al., 2013). The increase in response delays and not least variability of delays under anesthesia will profoundly affect the temporal structure and impair the efficiency of the network such as "binding" of the sensory image during cortical processing (Engel and Singer, 2001).

The increased response latency with anesthesia is in accordance with Pisauro et al. (2013) and Durand et al. (2016), recording multiunit-activity (MUA) and single unit data from separate animals where analyses are conducted on separate pools of units. Pisauro et al. (2013) report a peak latency of $\sim 92 \mathrm{~ms}$ in awake V1 mouse in MUA recordings, and $\sim 189 \mathrm{~ms}$ in anesthesia (two regimes: urethane and Isoflurane), while Durand et al. (2016) report $\sim 97 \mathrm{~ms}$ in awake mouse V1 single units and $\sim 120$ $\mathrm{ms}$ in a separate anesthesia population (urethane). These numbers are comparable to our latency measure of awake ( $\sim 105 \mathrm{~ms})$ and anesthesia ( $\sim 153 \mathrm{~ms})$. Our results verify these findings and elaborate by showing how individual units in a population increase their stimulus-evoked latency at a varied degree between the states for both peak rates and peak onset (Fig. $2 I, K)$. In addition, the prolonged LFP response during anesthesia is comparable to that reported in Haider et al. (2013), where they show large differences in the dynamics of inhibition between the states.

Furthermore, we show how different anesthetics cause different unit response delays with the anesthetic regimes with a predominantly GABAergic agonistic action having the largest impact on latencies (Fig. 2L). Wang et al. (2014) found that a synchronized state (anesthesia) is associated with longer latency in visual cortical neurons. They argue that it is not the anesthetic state itself, but rather the synchrony that is coupled to increases in latency. However, in their study they manipulate the presence of the synchronized state with on/off usage of Isoflurane on top of a stable urethane anesthesia. In light of the present findings and others (Cheung et al., 2001; Haider et al., 2013), it could be argued that it is the choice of anesthetic (the GABAergic action of Isoflurane) that is responsible for the increases in neuronal delay and not the synchronized state. Thus, the degree of latency observed in the current study and that of Wang et al. (2014) is caused by the use of GABAergic agonistic anesthetics. A smaller delay was indeed apparent in the Ketamine/ Xylazine condition.

When comparing findings from different primary cortical areas, it appears like the various cortical areas are influenced differently by anesthesia. While units in the somatosensory cortex of rabbits appear to show delays with anesthesia (Angel et al., 1973), much like our finding in the visual cortex, units in the auditory cortex appear to show the opposite result. A study by Ter-Mikaelian et al. (2007) on the Mongolian gerbil shows that the response to tones in auditory units is faster under anesthesia than in the awake state. It is plausible that the choice of a nonGABAergic anesthetic in their study was responsible for this discrepancy. A previous investigation finds larger delays in auditory anesthetic responses of cats with the GABAergic agonistic Isoflurane compared to pentobarbital anesthesia (Cheung et al., 2001). However, the reported awake response time in Ter-Mikaelian study is much longer than the anesthetic regime that gave the longest delay (Isoflurane) in the Cheung study, while the pentobarbital response was comparable between the studies. Although comparing latency estimates from different investigations may be problematic due to varying criteria of what qualifies as an evoked response, similar latency values with a non-GABAergic anesthetic in the two experiments suggests a degree of validity to the comparison. Accordingly, awake response times appear to be delayed in auditory cortex compared to several forms of anesthetics, including the anesthetic responsible for the greatest delay in all visual studies (Isoflurane). This yields support to the conclusion that both cortical area and anesthetic regime are determinants of the impact of anesthesia on the latency of units. 


\section{Pair-wise correlations increase under anesthesia}

The low pair-wise correlations of neurons in the sensory cortices may indicate the fraction of shared inputs (Alonso et al., 2001; Ko et al., 2011) rather than the direct connectivity between neurons (Moore et al., 1970). The increased pair-wise correlations in anesthesia compared with awake (Fig. 3) in our data are in accordance with recent findings that correlations may vary according to state of the animal (Greenberg et al., 2008; Ecker et al., 2010; Renart et al., 2010), suggesting that the structure of population activity depends on brain state. We supplement these findings by adding that the increase of pairwise correlations occurs both under stimulus-evoked and spontaneous events. This suggests that the synchronization induced by anesthesia occurs regardless of changing visual parameters and could likely reflect a general upregulating mechanism. This is in agreement with what has been reported on a larger scale across brain regions using fMRI and LFP (Bettinardi et al., 2015). We also find that spontaneous time periods in both states induce a higher pair-wise correlation between the units than during evoked, which implies that visual stimuli appear to desynchronize the activity of unit pairs, irrespective of state. A similar tendency for higher pair-wise correlations during spontaneous time periods versus evoked has been reported in the anesthetized monkey (Smith and Kohn, 2008). Furthermore, although a previous study describes a reduction in neuronal variability with sensory stimulation (Churchland et al., 2010), this comparison is across stimulus trials and does not reflect the correlation between units in a single trial. The result of Churchland et al. (2010) is therefore more applicable to our findings on the stability of visually evoked temporal sequences within states.

Furthermore, while pair-wise correlations returned to baseline levels $24 \mathrm{~h}$ after anesthesia, the correlations were significantly lower $30 \mathrm{~min}$ after animals recovered from anesthesia than the preanesthetic awake condition. This could indicate some desynchronizing mechanism during the recovery from anesthesia. Future investigations are needed to reveal the time course and synchrony of the recovery phase.

\section{Temporal sequences within and between states}

In several sensory systems, short-latency responses correlate with simple stimulus features, whereas later responses often represent more complex features. Our recordings from the same local populations in awake and anesthesia enabled direct comparisons of sequence in firing activity within a local population and across states. We first showed that within populations of recorded units in the visual cortex there is a clear temporal sequence of firing during specific visual stimulation that is preserved between awake recording sessions (Fig. 4). Moreover, we find a clear temporal structure within the populations in response to visual stimuli when comparing separate time points of anesthesia. Thus, units in the visual cortex appear to share the trait discovered by Luczak et al. (2007, 2009) from the auditory and somatosensory cortex. They reported that a sequence structure in firing activity within a population is retained across types of stimuli and occurs spontaneously during UP states, suggesting a stereotyped mode of information flow in a local cortical population (Luczak et al., 2007, 2009). They suggested that neurons firing earliest in the sequence would reflect an initial processing of incoming information; neurons firing at later time points would have access to the results of computations made by earlier firing ones and, thus, be capable of more sophisticated analysis.

While previous investigations were limited to comparing separate populations, we show that the temporal sequence of firing within a population is reduced in the transition between awake to anesthesia. We find this both in overall MSLs per unit and in the correlations of ranks between populations of units. This suggests a statedependent preservation of temporal firing structure within a population where the cortical state determines the local dynamics that produces the temporal firing sequence within the cortical microcircuit. This suggests less stereotypic temporal structure in V1 than previously suggested from findings in auditory and somatosensory cortex (Luczak et al., 2009). However, the partly preserved sequence of activity between awake and anesthesia suggests that some connections may be more hard-wired and independent of state.

\section{Increased delay with lower spatial frequencies in anesthesia}

We observed a preference toward higher spatial frequencies of $\mathrm{V} 1$ neurons in the awake state compared to anesthesia. This is accordance with preferred spatial frequencies during anesthesia in anesthetized rodents (Girman et al., 1999; Niell and Stryker, 2008; Gao et al., 2010) and an increases in spatial frequency preferences in awake mouse V1 (Andermann et al., 2011; Durand et al., 2016) and in awake versus anesthetized primate LGN (Alitto et al., 2011). However, while previous investigations compared responses in separate experiments, our chronic recordings allowed the detection of changes in single units recorded under anesthesia and in the awake state.

We find that for the highest spatial frequencies $(0.3 \mathrm{c} / \mathrm{d})$, it is mostly units in the awake state that respond (Fig. $5 B, C)$. When separating units on their preference toward low or high frequencies in the awake state, we found that the low preferring units remained mostly unchanged under anesthesia, while the high frequency group shifted their preference toward lower spatial frequencies in anesthesia (Fig. 5D). This shows that it is specifically units with high spatial frequency preference that change with the transition between awake to anesthesia and is not a result of a collective shift in all units toward lower preferences. High spatial frequencies may demand high temporal specificity and perhaps the prolonged evoked latencies and disrupted firing sequences in anesthesia impair the efficiency of processing higher frequencies.

Finally, we show that changing the spatial frequency of the visual stimulus induces differing response-latencies in anesthesia and not in the awake state (Fig. 5G). Visual stimuli consisting of drifting gratings with a broader width (low spatial frequency) produces more prolonged latencies in anesthesia compared to stimuli with a more narrow 
Table 2. Statistical description of all tests

\begin{tabular}{|c|c|c|c|}
\hline Comparison & Data structure & Type of test & Power or confidence interval (lower/upper 95\%) \\
\hline $\begin{array}{l}\text { Figure } 1 B \\
\text { awake-anesthesia-recovery }\end{array}$ & Non-normal & $\begin{array}{l}\text { Friedman's and Dunn's } \\
\text { post hoc }\end{array}$ & $\begin{array}{l}\text { Awake Cl: } 2.74 / 3.83 \\
\text { Anesthesia Cl: } 0.90 / 1.41 \\
\text { Recovery Cl: } 2.41 / 3.54\end{array}$ \\
\hline Figure $1 E$, active-sessile-anesthesia & Non-normal & $\begin{array}{l}\text { Friedman's and Dunn's } \\
\text { post hoc }\end{array}$ & $\begin{array}{l}\text { Active } \mathrm{Cl}: 3.32 / 5.39 \\
\text { Sessile } \mathrm{Cl}: 2.93 / 4.89 \\
\text { Anesthesia } \mathrm{Cl}: 1.48 / 3.47\end{array}$ \\
\hline $\begin{array}{l}\text { Figure } 1 G \text {, narrow spiking firing rates } \\
\text { awake-anesthesia }\end{array}$ & Non-normal & $\begin{array}{l}\text { Wilcoxon signed ranks } \\
\text { test }\end{array}$ & $\begin{array}{l}\text { Awake } \mathrm{Cl}: 4.38 / 9.21 \\
\text { Anesthesia } \mathrm{Cl}: 0.81 / 3.29\end{array}$ \\
\hline $\begin{array}{l}\text { Figure } 1 G \text {, broad spiking firing rates } \\
\text { awake-anesthesia }\end{array}$ & Non-normal & $\begin{array}{l}\text { Wilcoxon signed ranks } \\
\text { test }\end{array}$ & $\begin{array}{l}\text { Awake Cl: } 2.23 / 2.88 \\
\text { Anesthesia Cl: 0.85/1.29 }\end{array}$ \\
\hline $\begin{array}{c}\text { Figure } 1 G, \text { NS aw-an difference } \\
\text { versus BS aw-an difference }\end{array}$ & Non-normal & Mann-Whitney $U$ test & $\begin{array}{l}\text { BS diff Cl: } 1.16 / 1.81 \\
\text { NS diff Cl: } 2.54 / 6.95\end{array}$ \\
\hline Figure $1 H$, awake-Isoflurane & Non-normal & $\begin{array}{l}\text { Wilcoxon signed ranks } \\
\text { test }\end{array}$ & $\begin{array}{l}\text { Awake Cl: 2.92/4.72 } \\
\text { Iso Cl: 0.60/1.41 }\end{array}$ \\
\hline $\begin{array}{l}\text { Figure } 1 \mathrm{H}, \\
\text { awake-Isoflurane/Dormicum }\end{array}$ & Non-normal & $\begin{array}{l}\text { Wilcoxon signed ranks } \\
\text { test }\end{array}$ & $\begin{array}{l}\text { Awake Cl: } 2.51 / 3.30 \\
\text { Iso/Dor } \mathrm{Cl}: 0.74 / 1.28\end{array}$ \\
\hline Figure $1 H$, awake-Ketamine/Xylazine & Non-normal & $\begin{array}{l}\text { Wilcoxon signed ranks } \\
\text { test }\end{array}$ & $\begin{array}{l}\text { Awake Cl: } 1.78 / 3.27 \\
\text { Ket/Xyl Cl: } 1.75 / 4.02\end{array}$ \\
\hline $\begin{array}{l}\text { Figure } 1 H \text {, aw-an difference between } \\
\text { anesthetics }\end{array}$ & Non-normal & $\begin{array}{l}\text { Kruskal-Wallis and } \\
\text { Dunn's post hoc }\end{array}$ & $\begin{array}{l}\text { Iso/Dor diff } \mathrm{Cl}: 1.26 / 2.18 \\
\text { Iso diff } \mathrm{Cl}: 1.95 / 3.68 \\
\text { Ket/Xyl diff } \mathrm{Cl}:-1.16 / 0.44\end{array}$ \\
\hline $\begin{array}{l}\text { Figure } 1 \mathrm{~J} \text {, firing rate stability over } 1 \\
\mathrm{~h} \text {, between anesthetics }\end{array}$ & Non-normal & $\begin{array}{l}\text { Kruskal-Wallis and } \\
\text { Dunn's post hoc }\end{array}$ & $\begin{array}{l}\text { Iso/Dor diff } \mathrm{Cl}:-0.08 / 0.38 \\
\text { Iso diff } \mathrm{Cl}: 0.95 / 2.66 \\
\text { Ket/Xyl diff } \mathrm{Cl}:-1.00 /-0.08\end{array}$ \\
\hline Figure $1 K$, index of spontaneous and & Normal & Paired $t$ test & Ev/sp index awake-ev/sp index anest $\mathrm{Cl}:-0.20$ \\
\hline
\end{tabular}

Figure $1 K$, index of spontaneous and Normal Paired $t$ test evoked activity compared between awake and anesthesia

Figure $1 M$, evoked-spontaneous index change between awake and the different anesthetics

Figure $1 L$, comparison of spontaneous aw/spontaneous an index with evoked aw/evoked an index

Evoked/spontaneous index comparison between moving, sessile, and anesthesia

Figure $2 B$, visually evoked trough latency (LFP)

Figure $2 B$, visually evoked peak latency (LFP)

Figure $2 C$, comparison of the difference between trough and peak latency (LFP) in awake and anesthesia

Figure $2 D$, peak amplitude awake-anesthesia

Figure $2 \mathrm{~J}$, first evoked peak latency for single units, comparison aw-an

Figure $2 \mathrm{~J}$, first evoked peak latency onset for single units, comparison aw-an

Figure 2l, correlation latency aw-an

Figure $2 L$, latency anesthetic regimes; comparison of aw-an

Figure $2 L$, latency anesthetic regimes; comparison between regimes

Figure $3 B$, pair-wise CCs aw-an-re
Iso/Dor diffs: Non-normal Iso diffs: Normal Ket/Xyl diffs: Normal Non-normal

Non-normal

Non-normal

Non-normal

Non-normal

Non-normal

Non-normal

Non-normal

Non-normal

Iso/Dor and Iso: non-normal

Ket/Xyl: normal

Non-normal

Non-normal
Iso/Dor: Wilcoxon

Iso and Ket/Xyl: paired $t$ test

Wilcoxon signed ranks test

Friedman's and Dunn's post hoc

Wilcoxon signed ranks test

Wilcoxon signed ranks test

Wilcoxon signed ranks test

Wilcoxon signed ranks test

Wilcoxon signed ranks test

Wilcoxon signed ranks test

Spearman's

Iso/Dor and Iso:

Wilcoxon signed

ranks test

Ket/Xyl: paired $t$ test

Kruskal-Wallis and

Dunn's post hoc

Friedman's and Dunn's post hoc
Iso/Dor diff Cl: $-0.29 /-0.17$

Iso diff $\mathrm{Cl}$ : $-0.18 /-0.05$

Ket/Xyl diff Cl: $-0.14 / 0.01$

Spont Aw/Spont An index Cl: 0.49/0.59

Evoked Aw/Evoked An index Cl: 0.27/0.36

Mov-Sess ev/sp Cl: $-0.03 / 0.08$

Mov-Anest ev/sp Cl: $-0.20 /-0.05$

Sess-Anest ev/sp Cl: $-0.24 /-0.06$

Aw Trough Cl: 0.13/0.14

An Trough Cl: 0.14/0.17

Aw peak Cl: 0.19/0.24

An peak Cl: $0.28 / 0.42$

Aw trough-peak Cl: 0.05/0.10

An trough-peak Cl: 0.15/0.26

Aw peak amp Cl: 102/183.5

An peak amp Cl: $34 / 73.37$

Aw peak Cl: 96.69/112.5

An peak Cl:141.4/164.7

Aw peak Cl: 63.34/87.66

An peak Cl:93.25/116.6

Latency diff aw-an Cl: 35/61.86

Diff aw-IsoDor Cl: 54.02/92.27

Diff aw-Iso Cl: $-3.15 / 46.93$

Diff aw-KetXyl Cl: $-4.13 / 45.32$

Diff aw-IsoDor Cl: 54.02/92.27

Diff aw-Iso Cl: $-3.15 / 46.93$

Diff aw-KetXyl Cl: $-4.13 / 45.32$

CC Aw Cl: 0.008/0.012

CC An Cl:0.027/0.034

CC Re Cl: 0.004/0.008

(Continued) 
Table 2. Continued

\begin{tabular}{|c|c|c|c|}
\hline Comparison & Data structure & Type of test & Power or confidence interval (lower/upper 95\%) \\
\hline $\begin{array}{l}\text { Figure } 3 C \text {, pair-wise } C C \text { aw-an } \\
\text { correlation }\end{array}$ & Non-normal & $\begin{array}{l}\text { Spearman's rank } \\
\text { correlation }\end{array}$ & $\begin{array}{l}\text { Aw Cl: } 0.008 / 0.011 \\
\text { An Cl: } 0.023 / 0.028\end{array}$ \\
\hline Figure $3 B$, pair-wise $C C$ aw-an-re1 & Non-normal & $\begin{array}{l}\text { Friedman's and Dunn's } \\
\text { post hoc }\end{array}$ & $\begin{array}{l}\text { CC Aw Cl: 0.008/0.013 } \\
\text { CC An Cl: 0.029/0.036 } \\
\text { CC Re1 Cl: } 0.004 / 0.008\end{array}$ \\
\hline Figure $3 B$, pair-wise $C C$ aw-an-re2 & Non-normal & $\begin{array}{l}\text { Friedman's and Dunn's } \\
\text { post hoc }\end{array}$ & $\begin{array}{l}\text { CC Aw Cl: } 0.007 / 0.012 \\
\text { CC An Cl: } 0.027 / 0.035 \\
\text { CC Re2 Cl: } 0.004 / 0.009\end{array}$ \\
\hline $\begin{array}{l}\text { Figure } 3 D \text {, pair-wise } C C \text { evoked } \\
\text { aw-an }\end{array}$ & Non-normal & $\begin{array}{l}\text { Wilcoxon signed ranks } \\
\text { test }\end{array}$ & $\begin{array}{l}\text { CC Aw Ev Cl: 0.023/0.027 } \\
\text { CC An Ev Cl: 0.030/0.035 }\end{array}$ \\
\hline $\begin{array}{l}\text { Figure } 3 D \text {, pair-wise CC } \\
\text { Spontaneous aw-an }\end{array}$ & Non-normal & $\begin{array}{l}\text { Wilcoxon signed ranks } \\
\text { test }\end{array}$ & $\begin{array}{l}\text { CC Aw Sp Cl: 0.027/0.032 } \\
\text { CC An Sp Cl: 0.037/0.046 }\end{array}$ \\
\hline $\begin{array}{l}\text { Figure } 3 D \text {, pair-wise CC awake } \\
\text { ev-sp }\end{array}$ & Non-normal & $\begin{array}{l}\text { Wilcoxon signed ranks } \\
\text { test }\end{array}$ & $\begin{array}{l}\text { CC Aw Ev Cl: 0.023/0.027 } \\
\text { CC Aw Sp Cl: 0.027/0.032 }\end{array}$ \\
\hline $\begin{array}{l}\text { Figure } 3 D \text {, pair-wise } C C \text { anesthesia } \\
\text { ev-sp }\end{array}$ & Non-normal & $\begin{array}{l}\text { Wilcoxon signed ranks } \\
\text { test }\end{array}$ & $\begin{array}{l}\text { CC An Ev Cl: 0.030/0.035 } \\
\text { CC An Sp Cl: 0.037/0.046 }\end{array}$ \\
\hline Figure $3 E$, pair-wise CC Aw-BS & Non-normal & $\begin{array}{l}\text { Wilcoxon signed ranks } \\
\text { test }\end{array}$ & $\begin{array}{l}\text { CC Aw Cl: 0.005/0.011 } \\
\text { CC BS Cl: 0.040/0.052 }\end{array}$ \\
\hline Figure $3 E$, pair-wise CC Aw-non-BS & Non-normal & $\begin{array}{l}\text { Wilcoxon signed ranks } \\
\text { test }\end{array}$ & $\begin{array}{l}\text { CC Aw Cl: 0.008/0.016 } \\
\text { CC Non-BS Cl:0.023/0.036 }\end{array}$ \\
\hline $\begin{array}{l}\text { Figure } 3 E \text {, pair-wise CC Diff Aw-BS } \\
\text { vs Aw-non-BS }\end{array}$ & Non-normal & $\begin{array}{l}\text { Wilcoxon signed ranks } \\
\text { test }\end{array}$ & $\begin{array}{l}\text { CC Diff Aw-BS Cl: }-0.043 /-0.033 \\
\text { CC Diff Aw-NonBS Cl: }-0.022 /-0.013\end{array}$ \\
\hline $\begin{array}{l}\text { Figure } 3 F \text {, pair-wise CC across } \\
\text { anesthetic regimes }\end{array}$ & Non-normal & $\begin{array}{l}\text { Kruskal-Wallis and } \\
\text { Dunn's post hoc }\end{array}$ & $\begin{array}{l}\text { CC Aw-Iso Cl: }-0.036 /-0.027 \\
\text { CC Aw-Ket/Xyl Cl:-0.010/-0.005 } \\
\text { CC Aw Iso/Dor Cl:-0.012/-0.005 }\end{array}$ \\
\hline $\begin{array}{l}\text { Figure } 3 G, C V \text { in units in awake and } \\
\text { anesthesia }\end{array}$ & Non-normal & $\begin{array}{l}\text { Wilcoxon signed ranks } \\
\text { test }\end{array}$ & $\begin{array}{l}\text { CV Awake Cl: 1.16/1.24 } \\
\text { CV Anest Cl: 1.04/1.14 }\end{array}$ \\
\hline $\begin{array}{l}\text { Figure } 3 H \text {, CV within Aw-Iso, Aw-Iso/ } \\
\text { Dor, Aw-Ket/Xyl }\end{array}$ & Non-normal & $\begin{array}{l}\text { Wilcoxon signed ranks } \\
\text { tests }\end{array}$ & $\begin{array}{l}\text { CV Aw-iso diff Cl: }-0.12 / 0.14 \\
\text { CV Aw-iso/dor diff } \mathrm{Cl}:-0.20 /-0.05 \\
\text { CV Aw-ket/xyl diff } \mathrm{Cl}:-0.35 /-0.11\end{array}$ \\
\hline $\begin{array}{l}\text { Figure } 3 H, C V \text { between anesthetic } \\
\text { regimes }\end{array}$ & Non-normal & $\begin{array}{l}\text { Kruskal-Wallis and } \\
\text { Dunn's post hoc }\end{array}$ & $\begin{array}{l}\text { CV Aw-iso diff } \mathrm{Cl}:-0.12 / 0.14 \\
\text { CV Aw-iso/dor diff } \mathrm{Cl}:-0.20 /-0.05 \\
\text { CV Aw-ket/xyl diff } \mathrm{Cl}:-0.35 /-0.11\end{array}$ \\
\hline $\begin{array}{l}\text { Figure } 4 A \text {, MSL correlation between } \\
\text { states }\end{array}$ & Non-normal & $\begin{array}{l}\text { Spearman's rank } \\
\text { correlation }\end{array}$ & $\begin{array}{l}\text { Aw1-Aw2 Cl: 0.29/0.60 } \\
\text { An1-An2 Cl: 0.32/0.64 } \\
\text { Aw-An Cl: } 0.10 / 0.48\end{array}$ \\
\hline $\begin{array}{l}\text { Figure } 4 B \text {, single trial rank } \\
\text { correlations (CC); comparison aw- } \\
\text { aw, aw-an, an- an }\end{array}$ & Non-normal & $\begin{array}{l}\text { Kruskal-Wallis and } \\
\text { Dunn's post hoc }\end{array}$ & $\begin{array}{l}\text { Aw-Aw CC Cl: } 0.17 / 0.23 \\
\text { Aw-An CC Cl: } 0.06 / 0.13 \\
\text { An-An CC Cl: } 0.18 / 0.28\end{array}$ \\
\hline $\begin{array}{l}\text { Skewness test; } t \text { test to a theoretical } \\
\text { mean of zero on single trial rank } \\
\text { data }\end{array}$ & Normal & One-sample $t$ test & $\begin{array}{l}\text { Aw-Aw Cl: } 0.168 / 0.255 \\
\text { Aw-An Cl: } 0.065 / 0.148 \\
\text { An-An Cl: } 0.193 / 0.284\end{array}$ \\
\hline $\begin{array}{l}\text { CCs for individual populations of } \\
>15 \text { units followed between } \\
\text { states }\end{array}$ & Non-normal & $\begin{array}{l}\text { Spearman's rank } \\
\text { correlation }\end{array}$ & $\begin{array}{l}\text { Pop1: Aw-aw Cl: 0.50/0.94 } \\
\text { Aw-an Cl: }-0.42 / 0.62 \\
\text { Pop2: Aw-aw Cl: 0.44/0.90 } \\
\text { Aw-an Cl: }-0.34 / 0.62 \\
\text { An-an Cl:0.26/0.87 } \\
\text { Pop3: Aw-aw Cl: }-0.03 / 0.77 \\
\text { Aw-an Cl: }-0.06 / 0.77 \\
\text { An-an Cl: 0.24/0.87 } \\
\text { Pop4: Aw-aw Cl: } 0.05 / 0.80 \\
\text { Aw-an Cl: 0.32/0.88 } \\
\text { An-an Cl:0.25/0.86 }\end{array}$ \\
\hline $\begin{array}{l}\text { Skewness test; } t \text { test to a theoretical } \\
\text { mean of zero on single trial rank } \\
\text { shuffled data }\end{array}$ & Normal & One-sample $t$ test & $\begin{array}{l}\text { Aw-Aw Cl: }-0.023 / 0.066 \\
\text { Aw-An Cl: }-0.048 / 0.037 \\
\text { An-An Cl: }-0.066 / 0.027\end{array}$ \\
\hline $\begin{array}{l}\text { ANOVA on single trial rank corr } \\
\text { shuffle data }\end{array}$ & Normal & Continued) & $\begin{array}{l}\text { Aw-Aw Cl: }-0.023 / 0.066 \\
\text { Aw-An Cl: }-0.048 / 0.037 \\
\text { An-An Cl: }-0.066 / 0.027\end{array}$ \\
\hline
\end{tabular}


Table 2. Continued

\begin{tabular}{lll}
\hline Comparison & Data structure & Type of test \\
$\begin{array}{l}\text { Single trial rank correlations }(C C) \\
\text { versus shuffled units in all }\end{array}$ & Non-normal & Wilcoxon signed ranks \\
comparisons & & tests
\end{tabular}

Power or confidence interval (lower/upper 95\%)

Data Aw-aw CC Cl: 0.17/0.23

Shuffle Aw-aw CC Cl: $-0.012 / 0.05$

Data Aw-an CC Cl: 0.06/0.13

Shuffle Aw -an CC Cl:0.00/0.07

Data An-An CC Cl: 0.18/0.28

Figure 4C Rank by rank measure.

Normal

ANOVA and Tukey's post hoc test

CCs between states.

Figure $4 D$, single trial rank correlations (CC) for sessile-moving vs moving-anesthesia comparison Figure $5 B$, comparison of normalized responses to various spatial

frequencies

Figure 5D, correlation between awake and anesthetized normalized unit responses in units with the awake preference of low spatial frequencies

Figure $5 D$, correlation between awake and anesthetized normalized unit responses in units with the awake preference of high spatial frequencies

Figure $5 \mathrm{H}$, comparison of normalized responses to various temporal frequencies

Figure 5G, first peak latencies for units under different spatia frequencies

First peak delay between awake and anesthesia in all spatial frequencies

Figure 5J, first peak latencies for units under different temporal frequencies

First peak delay between awake and anesthesia in all spatial frequencies
Non-normal

Non-normal

$2 \mathrm{~Hz}$ : Normal

$4 \mathrm{~Hz}$ : Non-normal

$8 \mathrm{~Hz}$ : Normal

SF 0.02 vs $0.04,0.08,0.16$ and $0.3 \mathrm{c} / \mathrm{d}$ : Non-normal, Normal, Normal and Normal.

SF 0.04 vs $0.08,0.16$ and 0.3 $\mathrm{c} / \mathrm{d}$ : Normal, Normal and Normal.

SF 0.08 vs 0.16 and $0.3 \mathrm{c} / \mathrm{d}$ : Non-normal and Normal

SF 0.16 vs $0.16 \mathrm{c} / \mathrm{d}$ : Normal

SF 0.02 c/d: Normal

SF $0.04 \mathrm{c} / \mathrm{d}$ : Non-normal

SF $0.08 \mathrm{c} / \mathrm{d}$ : Non-normal

SF $0.16 \mathrm{c} / \mathrm{d}$ : Non-normal

SF $0.3 \mathrm{c} / \mathrm{d}$ : Normal

TF 2 vs 4 and $8 \mathrm{~Hz}$ :

Non-normal and Normal

TF 4 vs $8 \mathrm{~Hz}$ : Normal

TF $2 \mathrm{~Hz}$ :Normal

TF $4 \mathrm{~Hz}$ : Non-normal

TF $8 \mathrm{~Hz}$ : Normal
Mann-Whitney $U$ test

Wilcoxon signed ranks tests

Spearman's rank correlation

Spearman's rank correlation

Paired $t$ test and Wilcoxon signed ranks tests

Paired $t$ test and Wilcoxon signed ranks tests

Paired $t$ tests and Wilcoxon signed ranks tests

Paired $t$ tests and Wilcoxon signed ranks test

Paired $t$ tests and Wilcoxon signed ranks test
Shuffle An-an CC Cl: $-0.07 / 0.02$

CC Aw-aw Cl: 0.28/0.67

CC Aw-an Cl: $-0.41 / 0.38$

CC Aw-an Cl: 0.51/0.74

SessMov Cl: 0.094/0.177

MovAnest Cl: 0.032/0.106

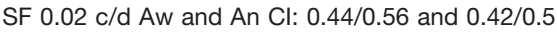
SF $0.04 \mathrm{c} / \mathrm{d}$ Aw and $\mathrm{An} \mathrm{Cl:} 0.47 / 0.57$ and $0.42 / 0.54$

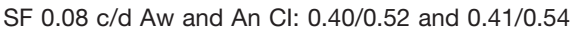
SF $0.16 \mathrm{c} / \mathrm{d}$ Aw and $\mathrm{An} \mathrm{Cl:} 0.36 / 0.48$ and $0.31 / 0.42$

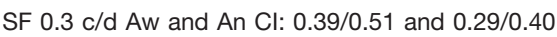
Low SF pref $\mathrm{Cl}$ : 0.23/0.61

High SF pref $\mathrm{Cl}:-0.31 / 0.15$

TF $2 \mathrm{~Hz}$ Aw and $\mathrm{An} \mathrm{Cl:} \mathrm{0.50/0.60} \mathrm{and} \mathrm{0.45/0.56}$

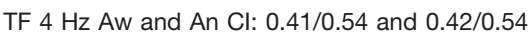
TF $8 \mathrm{~Hz}$ Aw and $\mathrm{An} \mathrm{Cl:} \mathrm{0.34/0.47} \mathrm{and} \mathrm{0.34/0.48}$ SF 0.02 c/d Aw and An Cl: 99.1/136.3 and 179.6/231.6 SF 0.04 c/d Aw and An Cl: 104.7/134.7 and

$181.6 / 222.6$

SF 0.08 c/d Aw and An Cl: 92.15/121 and 158.2/205.9

SF $0.16 \mathrm{c} / \mathrm{d}$ Aw and $\mathrm{An} \mathrm{Cl:} \mathrm{98.27/136.6} \mathrm{and}$

144.6/194.2

SF 0.3 c/d Aw and An Cl: 95.34/129.2 and 126.6/181.6

SF 0.02 c/d Diff Cl: 59.8/113.8

SF 0.04 c/d Diff Cl: 53.57/110.1

SF 0.08 c/d Diff Cl: 43.25/104

SF 0.16 c/d Diff Cl: 26.28/88.95

SF $0.3 \mathrm{c} /$ d Diff Cl: 5.42/73.04

TF $2 \mathrm{~Hz}$ Aw and $\mathrm{An} \mathrm{Cl:} \mathrm{91.22/135.9} \mathrm{and} \mathrm{159.6/211.8}$

TF $4 \mathrm{~Hz} \mathrm{Aw}$ and $\mathrm{An} \mathrm{Cl:} \mathrm{84.6/117.6} \mathrm{and} \mathrm{155.6/203.8}$

TF $8 \mathrm{~Hz}$ Aw and $\mathrm{An} \mathrm{Cl:} \mathrm{84.88/122.5} \mathrm{and} \mathrm{149.5/194.7}$

TF 2 Hz Diff Cl: 43.59/104.4

TF 4 Hz Diff Cl: 41.04/92.87

TF $8 \mathrm{~Hz}$ Diff Cl: 34.64/101.9 width (high spatial frequency). Such a change may be expected due to the slower increase of contrast in each unit's receptive field during low spatial frequency conditions. However, the absence of this change in the awake states suggest a difference in the processing of these stimuli in the two states. The unit latency under all spatial frequencies in the awake state is surprisingly similar. In light of the findings by Haider et al. (2013) of increased and faster inhibition in the awake state, it is possible that fast inhibition terminates the more prolonged responses found in anesthesia under lower spatial frequencies. Thus, if responses are inhibited and terminated in the awake state, this may conceal the response differences found in anesthesia. The finding that awake inhibition dominates sensory processing while GABAergic agonistic anesthesias induce delays and alterations in temporal responses may be counterintuitive. However, since we also find that putative fast-spiking interneurons have a larger firing rate decrease compared to the general population, this suggests that their ability to regulate/inhibit activity is diminished. Fast spiking inhibitory neurons indeed have a higher GABAergic receptor density (Klausberger et al., 2002), allowing a direct agonistic action from the anesthetics. It can be hypothesized that it is the potentially 
direct influence of anesthetics on fast-spiking inhibitory neurons that remove the large and fast inhibition of responses to the varying spatial frequencies that is present in the awake state.

In addition, we observe with interest that many of salient features of the observed differences between awake and anesthetized states are reproduced qualitatively by the relatively simple Brunel-type model networks when the anesthetic actions are assumed to increase inhibition in the network (Fig. 6). In systems neuroscience such a model-based analysis approach where candidate network models are compared quantitatively with experimental data, is still in its infancy, primarily due to the lack of candidate network models amenable for such analysis (but see Potjans and Diesmann, 2014; Hagen et al., 2016). As more such models become available, we expect the present workflow for comparing experiments with models to become more prevalent.

\section{References}

Alitto HJ, Moore BD, Rathbun DL, Usrey WM (2011) A comparison of visual responses in the lateral geniculate nucleus of alert and anaesthetized macaque monkeys. J Physiol 589:87-99. CrossRef Medline

Alonso JM, Usrey WM, Reid RC (2001) Rules of connectivity between geniculate cells and simple cells in cat primary visual cortex. J Neurosci 21:4002-4015. Medline

Andermann ML, Kerlin AM, Roumis DK, Glickfeld LL, Reid RC (2011) Functional specialization of mouse higher visual cortical areas. Neuron 72:1025-1039. CrossRef Medline

Angel A, Berridge D, Unwin J (1973) The effect of anaesthetic agents on primary cortical evoked responses. Brit J Anaesth 45:824-836. Medline

Armstrong-James M, George MJ (1988) Influence of anesthesia on spontaneous activity and receptive field size of single units in rat Sm1 neocortex. Exp Neurol 99:369-387. Medline

Ayaz A, Saleem AB, Schölvinck ML, Carandini M (2013) Locomotion controls spatial integration in mouse visual cortex. Curr Biol 23: 890-894. CrossRef Medline

Barthó P, Hirase H, Monconduit L, Zugaro M, Harris KD, Buzsáki G (2004) Characterization of neocortical principal cells and interneurons by network interactions and extracellular features. J Neurophysiol 92:600-608. CrossRef Medline

Bayer H (2008) Awake and asleep. Nat Neurosci 11:740. CrossRef Medline

Bettinardi RG, Tort-Colet N, Ruiz-Mejias M, Sanchez-Vives MV, Deco G (2015) Gradual emergence of spontaneous correlated brain activity during fading of general anesthesia in rats: evidences from fMRI and local field potentials. Neuroimage 114:185-198. CrossRef Medline

Brainard DH (1997) The psychophysics toolbox. Spat Vis 10:433436. Medline

Brincat SL, Connor CE (2006) Dynamic shape synthesis in posterior inferotemporal cortex. Neuron 49:17-24. CrossRef Medline

Brunel N (2000) Dynamics of sparsely connected networks of excitatory and inhibitory spiking neurons. J Comput Neurosci 8:183208. Medline

Bruno RM, Simons DJ (2002) Feedforward mechanisms of excitatory and inhibitory cortical receptive fields. J Neurosci 22:1096610975.

Buzsáki G (2006) Rhythms of the brain. New York: Oxford University Press.

Cheung SW, Nagarajan SS, Bedenbaugh PH, Schreiner CE, Wang X, Wong A (2001) Auditory cortical neuron response differences under isoflurane versus pentobarbital anesthesia. Hear Res 156:115127. Medline
Churchland MM, Yu BM, Cunningham JP, Sugrue LP, Cohen MR, Corrado GS, Newsome WT, Clark AM, Hosseini P, Scott BB, Bradley DC, Smith MA, Kohn A, Movshon JA, Armstrong KM, Moore T, Chang SW, Snyder LH, Lisberger SG, Priebe NJ, et al. (2010) Stimulus onset quenches neural variability: a widespread cortical phenomenon. Nat Neurosci 13:369-378. CrossRef Medline

Cimenser A, Purdon PL, Pierce ET, Walsh JL, Salazar-Gomez AF, Harrell PG, Tavares-Stoeckel C, Habeeb K, Brown EN (2011) Tracking brain states under general anesthesia by using global coherence analysis. Proc Natl Acad Sci USA 108:8832-8837. CrossRef Medline

Csicsvari J, Hirase H, Czurkó A, Mamiya A, Buzsáki G (1999) Oscillatory coupling of hippocampal pyramidal cells and interneurons in the behaving Rat. J Neurosci 19:274-287.

Durand S, lyer R, Mizuseki K, de Vries S, Mihalas S, Reid RC (2016) A comparison of visual response properties in the lateral geniculate nucleus and primary visual cortex of awake and anesthetized mice. J Neurosci 36:12144-12156. CrossRef Medline

Dyer RS, Rigdon GC (1987) Urethane affects the rat visual system at subanesthetic doses. Physiol Behav 41:327-330. Medline

Ecker AS, Berens P, Keliris GA, Bethge M, Logothetis NK, Tolias AS (2010) Decorrelated neuronal firing in cortical microcircuits. Science 327:584-587. CrossRef Medline

Edwards FA, Konnerth A, Sakmann B (1990) Quantal analysis of inhibitory synaptic transmission in the dentate gyrus of rat hippocampal slices: a patch-clamp study. J Physiol 430:213-249. Medline

Engel AK, Singer W (2001) Temporal binding and the neural correlates of sensory awareness. Trends Cogn Sci 5:16-25. Medline

Fee MS, Mitra PP, Kleinfeld D (1996) Automatic sorting of multiple unit neuronal signals in the presence of anisotropic and nonGaussian variability. J Neurosci Methods 69:175-188. CrossRef Medline

Ferron JF, Kroeger D, Chever O, Amzica F (2009) Cortical inhibition during burst suppression induced with isoflurane anesthesia. $J$ Neurosci 29:9850-9860. CrossRef Medline

Gao E, DeAngelis GC, Burkhalter A (2010) Parallel input channels to mouse primary visual cortex. J Neurosci 30:5912-5926. CrossRef Medline

Gasparini S, Magee JC (2006) State-dependent dendritic computation in hippocampal CA1 pyramidal neurons. J Neurosci 26:20882100. CrossRef Medline

Gewaltig M-O, Diesmann M (2007) NEST (neural simulation tool). Scholarpedia 2:1430. CrossRef

Girman SV, Sauvé Y, Lund RD (1999) Receptive field properties of single neurons in rat primary visual cortex. J Neurophysiol 82:301311. Medline

Greenberg DS, Houweling AR, Kerr JND (2008) Population imaging of ongoing neuronal activity in the visual cortex of awake rats. Nat Neurosci 11:749-751. CrossRef Medline

Hagen E, Dahmen D, Stavrinou ML, Lindén H, Tetzlaff T, van Albada SJ, Grün S, Diesmann M, Einevoll GT (2016) Hybrid scheme for modeling local field potentials from point-neuron networks. Cereb Cortex 26:4461-4496. CrossRef Medline

Haider B, Häusser M, Carandini M (2013) Inhibition dominates sensory responses in the awake cortex. Nature 493:97-100. CrossRef Medline

Harris KD, Henze DA, Csicsvari J, Hirase H, Buzsáki G (2000) Accuracy of tetrode spike separation as determined by simultaneous intracellular and extracellular measurements. J Neurophysiol 84: 401-414. Medline

Hentschke H, Schwarz C, Antkowiak B (2005) Neocortex is the major target of sedative concentrations of volatile anaesthetics: strong depression of firing rates and increase of GABAA receptormediated inhibition. Eur J Neurosci 21:93-102. CrossRef Medline Henze DA, Borhegyi Z, Csicsvari J, Mamiya A, Harris KD, Buzsáki G (2000) Intracellular features predicted by extracellular recordings in the hippocampus in vivo. J Neurophysiol 84:390-400. Medline 
Hofer SB, Ko H, Pichler B, Vogelstein J, Ros H, Zeng H, Lein E, Lesica NA, Mrsic-Flogel TD (2011) Differential connectivity and response dynamics of excitatory and inhibitory neurons in visual cortex. Nat Neurosci 14:1045-1052. CrossRef Medline

Hubel DH, Wiesel TN (1962) Receptive fields, binocular interaction and functional architecture in the cat's visual cortex. J Physiol 160:106-154. Medline

Hubel DH, Wiesel TN (1968) Receptive fields and functional architecture of monkey striate cortex. J Physiol 195:215-243. Medline

lurilli G, Ghezzi D, Olcese U, Lassi G, Nazzaro C, Tonini R, Tucci V, Benfenati F, Medini P (2012) Sound-driven synaptic inhibition in primary visual cortex. Neuron 73:814-828. CrossRef Medline

lurilli G, Olcese U, Medini P (2013) Preserved excitatory-inhibitory balance of cortical synaptic inputs following deprived eye stimulation after a saturating period of monocular deprivation in rats. PLoS One 8. CrossRef

Iyer R, Menon V, Buice M, Koch C, Mihalas S (2013) The influence of synaptic weight distribution on neuronal population dynamics. PLoS Comput Biol 9. CrossRef

Keller GB, Bonhoeffer T, Hübener M (2012) Sensorimotor mismatch signals in primary visual cortex of the behaving mouse. Neuron 74:809-815. CrossRef Medline

Kerr JND, Greenberg D, Helmchen F (2005) Imaging input and output of neocortical networks in vivo. Proc Natl Acad Sci USA 102: 14063-14068. CrossRef Medline

Klausberger T, Roberts JDB, Somogyi P (2002) Cell type- and inputspecific differences in the number and subtypes of synaptic GABA A receptors in the hippocampus. J Neurosci 22:2513-2521. Medline Medline

Ko H, Hofer SB, Pichler B, Buchanan KA, Sjöström PJ, Mrsic-Flogel TD (2011) Functional specificity of local synaptic connections in neocortical networks. Nature 473:87-91. CrossRef Medline

Kriener B, Helias M, Rotter S, Diesmann M, Einevoll GT (2014) How pattern formation in ring networks of excitatory and inhibitory spiking neurons depends on the input current regime. Front Comput Neurosci 7. CrossRef

Lee H, Simpson GV, Logothetis NK, Rainer G (2005) Phase locking of single neuron activity to theta oscillations during working memory in monkey extrastriate visual cortex. Neuron 45:147-156. CrossRef Medline

Lewis LD, Weiner VS, Mukamel EA, Donoghue JA, Eskandar EN, Madsen JR, Anderson WS, Hochberg LR, Cash SS, Brown EN, Purdon PL (2012) Rapid fragmentation of neuronal networks at the onset of propofol-induced unconsciousness. Proc Natl Acad Sci USA 109:E3377-E3386. CrossRef Medline

Luczak A, Barthó P, Marguet SL, Buzsáki G, Harris KD (2007) Sequential structure of neocortical spontaneous activity in vivo. Proc Natl Acad Sci USA 104:347-352. CrossRef Medline

Luczak A, Barthó P, Harris KD (2009) Spontaneous events outline the realm of possible sensory responses in neocortical populations. Neuron 62:413-425. CrossRef Medline

Luczak A, Bartho P, Harris KD (2013) Gating of sensory input by spontaneous cortical activity. J Neurosci 33:1684-1695. CrossRef Medline

Luczak A, MacLean JN (2012) Default activity patterns at the neocortical microcircuit level. Front Integr Neurosci 6. CrossRef

Maggi CA, Meli A (1986) Suitability of urethane anesthesia for physiopharmacological investigations in various systems Part 1: general considerations. Experientia 42:109-114. Medline

Mainen ZF, Sejnowski TJ (1995) Reliability of spike timing in neocortical neurons. Science 268:1503-1506. Medline

Mashour G (2014) Top-down mechanisms of anesthetic-induced unconsciousness. Front Syst Neurosci 8:115. CrossRef Medline

Mazze RI, Rice SA, Baden JM (1985) Halothane, isoflurane, and enflurane MAC in pregnant and nonpregnant female and male mice and rats. Anesthesiology 62:339-341. Medline

McCormick DA, Connors W, Lighthall W, Prince A (1985) Comparative electrophysiology of pyramidal and sparsely spiny stellate neurons of the neocortex. J Neurophysiol 54:782-806.
Moore GP, Segundo JP, Perkel DH, Levitan H (1970) Statistical signs of synaptic interaction in neurons. Biophysical Journa 10(9):876900. CrossRef

Niell CM, Stryker MP (2008) Highly selective receptive fields in mouse visual cortex. J Neurosci 28:7520-7536. CrossRef Medline

Niell CM, Stryker MP (2010) Modulation of visual responses by behavioral state in mouse visual cortex. Neuron 65:472-479. CrossRef

Pelli DG (1997) The VideoToolbox software for visual psychophysics: transforming numbers into movies. Spat Vis 10:437-442. Medline

Pisauro MA, Dhruv NT, Carandini M, Benucci A (2013) Fast hemodynamic responses in the visual cortex of the awake mouse. $J$ Neurosci 33:18343-18351. CrossRef Medline

Potjans TC, Diesmann M (2014) The cell-type specific cortical microcircuit: relating structure and activity in a full-scale spiking network model. Cereb Cortex 24:785-806. CrossRef

Raz A, Grady SM, Krause BM, Uhlrich DJ, Manning KA, Banks MI (2014) Preferential effect of isoflurane on top-down versus bottom-up pathways in sensory cortex. Front Syst Neurosci 8. CrossRef

Reinagel P, Reid RC (2002) Precise firing events are conserved across neurons. J Neurosci 22:6837-6841. Medline Medline

Renart A, Moreno-Bote R, Wang X-J, Parga N (2007) Mean-driven and fluctuation-driven persistent activity in recurrent networks. Neural Comput 19:1-46. CrossRef

Renart A, de la Rocha J, Bartho P, Hollender L, Parga N, Reyes A, Harris KD (2010) The asynchronous state in cortical circuits. Science 327:587-590. CrossRef Medline

Robbins AA, Fox SE, Holmes GL, Scott RC, Barry JM (2013) Short duration waveforms recorded extracellularly from freely moving rats are representative of axonal activity. Front Neural Circuits 7. CrossRef

Schneidman E, Berry MJ, Segev R, Bialek W (2006) Weak pairwise correlations imply strongly correlated network states in a neural population. Nature 440:1007-1012. CrossRef

Schulz DP, Carandini M (2010) An uncorrelated state for the cortex? F1000 Biol Rep 2:2-4.

Schummers J, Yu H, Sur M (2008) Tuned responses of astrocytes and their influence on hemodynamic signals in the visual cortex. Science 320:1638-1643. CrossRef Medline

Siapas AG, Lubenov EV, Wilson MA (2005) Prefrontal phase locking to hippocampal theta oscillations. Neuron 46:141-151. CrossRef Medline

Sirota A, Montgomery S, Fujisawa S, Isomura Y, Zugaro M, Buzsáki $\mathrm{G}$ (2008) Entrainment of neocortical neurons and gamma oscillations by the hippocampal theta rhythm. Neuron 60:683-697. CrossRef Medline

Skaggs WE, McNaughton BL, Wilson MA, Barnes CA (1996) Theta phase precession in hippocampal neuronal populations and the compression of temporal sequences. Hippocampus 6:149-172. CrossRef Medline

Sleigh JW, Vizuete JA, Voss L, Steyn-Ross A, Steyn-Ross M, Marcuccilli CJ, Hudetz AG (2009) The electrocortical effects of enflurane: experiment and theory. Anesth Analg 109:1253-1262. CrossRef Medline

Smith MA, Kohn A (2008) Spatial and temporal scales of neuronal correlation in primary visual cortex. J Neurosci 28:12591-12603. CrossRef Medline

Steriade M (2004) Neocortical cell classes are flexible entities. Nat Rev Neurosci 5:121-134. CrossRef Medline

Steriade M, Amzica F, Contreras D (1994) Cortical and thalamic cellular correlates of electroencephalographic burst-suppression. Electroencephalogr Clin Neurophysiol 90:1-16. Medline

Suner S, Fellows MR, Vargas-Irwin C, Nakata GK, Donoghue JP (2005) Reliability of signals from a chronically implanted, siliconbased electrode array in non-human primate primary motor cortex. IEEE Trans Neural Syst Rehabil Eng 13:524-541. CrossRef Medline 
Swank RL, Watson CW (1949) Effects of barbiturates and ether on spontaneous electrical activity of dog brain. J Neurophysiol 12: 137-160. Medline

Tallon-Baudry C, Bertrand O, Delpuech C, Permier J (1997) Oscillatory gamma-band $(30-70 \mathrm{~Hz})$ activity induced by a visual search task in humans. J Neurosci 17:722-734. Medline

Ter-Mikaelian M, Sanes DH, Semple MN (2007) Transformation of temporal properties between auditory midbrain and cortex in the awake Mongolian gerbil. J Neurosci 27:6091-6102. CrossRef Medline

Teramae JN, Fukai T (2014) Computational implications of lognormally distributed synaptic weights. Proc IEEE 102:500-512. CrossRef

Thrane AS, Thrane VR, Zeppenfeld D, Lou N, Xu Q, Nagelhus EA, Nedergaard M (2012) General anesthesia selectively disrupts astrocyte calcium signaling in the awake mouse cortex. Proc Natl Acad Sci USA 109:18974-18979. CrossRef

Tiesinga P, Fellous JM, Sejnowski TJ (2008) Regulation of spike timing in visual cortical circuits. Nat Rev Neurosci 9:97-107. CrossRef Medline

Vahle-Hinz C, Detsch O (2002) What can in vivo electrophysiology in animal models tell us about mechanisms of anaesthesia? $\mathrm{Br} J$ Anaesth 89:123-142. Medline
Van Aerde KI, Feldmeyer D (2015) Morphological and physiological characterization of pyramidal neuron subtypes in rat medial prefrontal cortex. Cereb Cortex 25:788-805. CrossRef Medline

Villeneuve MY, Casanova C (2003) On the use of isoflurane versus halothane in the study of visual response properties of single cells in the primary visual cortex. J Neurosci Methods 129:19-31. CrossRef

Vizuete JA, Pillay S, Diba K, Ropella KM, Hudetz AG (2012) Monosynaptic functional connectivity in cerebral cortex during wakefulness and under graded levels of anesthesia. Front Integr Neurosci 6. CrossRef

Wang X, Chen C, Zhang D, Yao H (2014) Cumulative latency advance underlies fast visual processing in desynchronized brain state. Proc Natl Acad Sci USA 111:515-520. CrossRef Medline

Wilson MA, McNaughton BL (1993) Dynamics of the hippocampal ensemble code for space. Science 261:1055-1058. Medline

Wurtz RH (1969) Visual receptive fields of striate cortex neurons in awake monkeys. J Neurophysiol 32:727-742. Medline

Zhang S, Xu M, Kamigaki T, Hoang Do JP, Chang W-C, Jenvay S, Miyamichi K, Luo L, Dan Y, Do J, Chang W-C, Jenvay S, Miyamichi K, Luo L, Dan Y (2014) Long-range and local circuits for top-down modulation of visual cortex processing. Science 345:660-664. CrossRef 